\title{
Eyes of covalent organic frameworks: cooperation between analytical chemistry and COFs
}

\author{
${ }^{1}$ Key Lab of Eco-Environments Related Polymer Materials of Ministry of Education, Key Lab of Bioelectrochemistry and En- \\ vironmental Analysis of Cansu Province, College of Chemistry and Chemical Engineering, Northwest Normal University, \\ Lanzhou 730070, P.R.China, E-mail: haogu012@126.com, xbsfda123@126.com \\ ${ }^{2}$ College of Chemistry and Chemical Engineering, Lanzhou City University, Provincial Key Laboratory of Cansu Higher Educa- \\ tion for City Environmental Pollution Control, Lanzhou City University, Lanzhou, Gansu Province 730070, P.R. China
}

\begin{abstract}
:
Covalent organic frameworks (COFs) are a class of porous organic crystal materials. Since Yaghi and co-workers reported the first COF material in 2005 (Côté, A. P.; Benin, A. I.; Ockwig, N. W.; O'Keeffe, M.; Matzger, A. J.; Yaghi, O. M. Science 2005, 310, 1166-1170), COFs have shown great potential and research value in many fields, for instance, gas storage and separation, photoelectric function, fluorescence sensor, catalysis, drug delivery, dye and pollutant adsorption, electronic devices and so on. In this review, the frequently used analysis and characterization methods for COFs are summarized, and two to three examples are selected for each analysis and characterization technique in order to explain it in detail. The selected examples of COFs, which either had a unique structure and feature, or were reported for the first time, can be conducive to understanding $\mathrm{COFs}$ and their analysis methods better and faster. The application of COFs in analysis and detection is also introduced in this review, including fluorescent analysis, separation and enrichment. The cooperation between analytical chemistry and COFs is fully reflected. This review is helpful for the person interested in this subject and provides some useful information on the characterization methods and the applications of COFs for the beginners.
\end{abstract}

Keywords: analytical chemistry, characterization, covalent organic frameworks, fluorescence, separation DOI: $10.1515 /$ revac-2017-0023

Received: March 26, 2017; Accepted: February 27, 2019

\section{Introduction}

People begun to use porous materials from the ancient times and there have had innumerable porous materials all over the world today. These kinds of materials can be divided into organic and inorganic, natural and synthetic. Classical porous materials include zeolites, activated carbon and metal organic framework compounds (Kitagawa, Kitaura, and Noro 2004). As porous materials, zeolite and activated carbon have been studied for a long time and widely used in petroleum processing, chemical industry and environmental protection and other fields (Davis and Lobo 1992 and Corma 1997) because of their abundant pores, large specific surface areas and surface acid-base properties. A novel material was reported in 1998 (Li et al. 1998), formed by the self-assembly of metal ions and organic ligands, called "metal-organic frameworks." Such materials have potential applications in various fields, such as optics (Rao et al. 2013), magnetics (Harbuzaru et al. 2008), chemical sensing (Kumar, Deep, and Kim 2015) and hydrogen storage (Yang et al. 2009). A variety of nanoscale porous organic polymers can be obtained by linking between different organic units with covalent bond, such as hypercross-linked polymers (Li et al. 2011, Luo et al. 2012), polymers of intrinsic microporosity (Budd et al. 2004 and McKeown and Budd 2006), conjugated microporous polymers (Jiang et al. 2007, Cooper 2009), porous aromatic framework (Ben et al. 2009) and so on. All these materials have high specific surface area and adjustable pore properties. In these organic porous materials, covalent organic framework (COF) is the special one. This material was reported by Professor Yaghi in 2005 for the first time, which was organic porous polymer with high crystallinity and covalent bond linking (Côté et al. 2005). This kind of materials is very important for the development of organic porous materials, which has many advantages such as porosity, light weight, high crystallinity, good stability, controllable pore diameter and designable function. They have been extensively 
studied in the past 10 years, and developed rapidly. Based on these studies, COFs show great potential and research value in many spheres, for instance, gas storage and separation (Graetz 2009, Sumida et al. 2012, Zou, Ren, and Zhu 2013, and Wang et al. 2016a), photoelectric function (Wan et al. 2008, Spitler and Dichtel 2010, Ding et al. 2011a, and Feng et al. 2012), fluorescence sensor (Wan et al. 2008, Zhang et al. 2012, Guo, Zeng, and Cao 2016, Lin et al. 2016, and Wang, Yang, and Yan 2018), catalysis (Ding et al. 2011a, Fang et al. 2014, Wang et al. 2014a, Xu et al. 2014, and Lin et al. 2017), drug delivery (Fang et al. 2015, Bai et al. 2016), dye and pollutant adsorption (Wang et al. 2014b, Liu et al. 2015, and Zhang, Li, and Zhang 2015), electronic devices (Ding et al. 2011a, Wan et al. 2011, and Chen et al. 2014) and so on.

Ding and Wang (2013) reported the building units that have been successfully utilized for the synthesis of COFs, and summarized six reaction types for synthesizing different COFs. Yaghi's group published a review in 2015; they introduced the COFs in the aspects of the crystallization, bonding, design and characterization, and properties and applications of COFs (Waller, Gandara, and Yaghi 2015). Significantly, more and more COFs have further been studied and developed for particular applications at present. With the rapid development of the modern instrumental analysis technique, the observation of COFs was more in-depth. Various analytical instruments are like "eyes"; the microscopic world can be observed in detail with the help of these "eyes." Moreover, in the efforts of chemists, COFs have been given a variety of functions, and they can be used for detecting and separating the target molecules. They are also the "eyes" of chemistry to observe the chemical world. Here, the methods of analysis and characterization of COFs in the published works are summarized and the applications of COFs in chemical detection are introduced.

\section{Analysis and characterization of COFs}

\subsection{Infrared spectrum}

COFs are mostly composed of $\mathrm{C}, \mathrm{H}, \mathrm{O}, \mathrm{N}, \mathrm{B}$ and other elements, linked by $\mathrm{C}-\mathrm{N}, \mathrm{C}=\mathrm{N}, \mathrm{C}=\mathrm{O}, \mathrm{B}-\mathrm{O}$ and other chemical bond (Waller, Gandara, and Yaghi 2015). Recently, it was reported that the S, P elements were used in COFs; these kinds of COFs present more diverse functions (Ding et al. 2016, Zhang et al. 2016a). The infrared (IR) spectrum is ordinarily used as an efficient method to evaluate the formation of these chemical bonds in the target compound. For example, the fourier transform infrared (FT-IR) spectra of COF-1, the first COF, constructed by condensation of 1,4-benzenediboronic acid (Figure 1) (Côté et al. 2005), indicated the formation of the expected boron-based ring groups in COF-1 and the generation of COF material. The result of IR can not only be used to determine the reaction to take place but also help us to know the extent of the reaction. Thomas and co-workers reported a covalent triazine-based framework (CTF) (Kuhn, Antonietti, and Thomas 2008); this COF was synthesized by the dynamic trimerization of aromatic nitriles (Figure 2). The FT-IR spectra of the products at different reaction times and temperatures performed the extent of the trimerization reactions. As the authors described, the trimerization reaction was followed by FT-IR measurements of the products at different reaction times. The disappearance of the intense carbonitrile band at $2218 \mathrm{~cm}^{-1}$ and the appearance of a strong absorption band at $1352 \mathrm{~cm}^{-1}$ were indicative of a successful trimerization reaction and formation of triazine rings (Manecke and Wöhrle 1968 and Kuhn, Antonietti, and Thomas 2008).
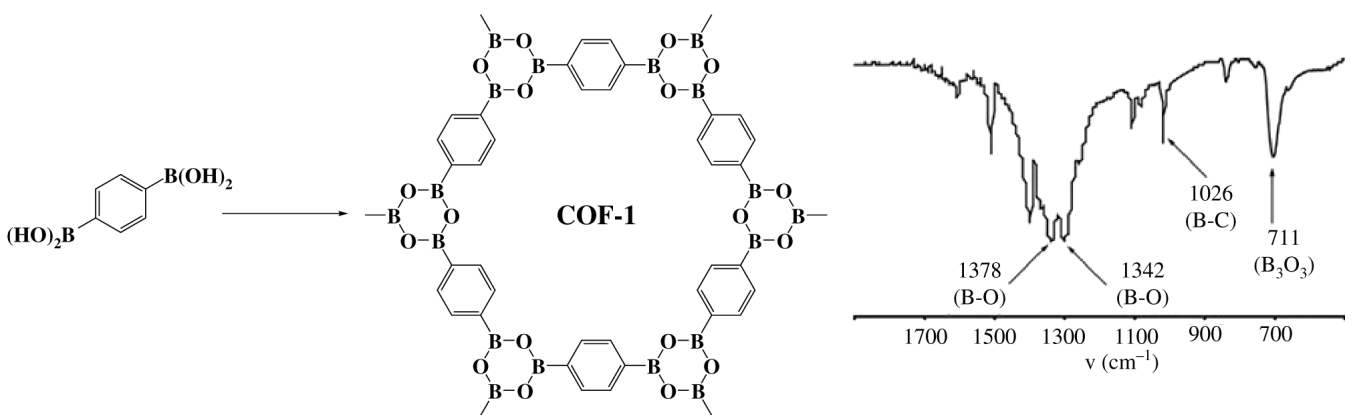

Figure 1: Condensation reactions of boronic acids used to produce COF-1 and the FT-IR spectra of COF-1. 

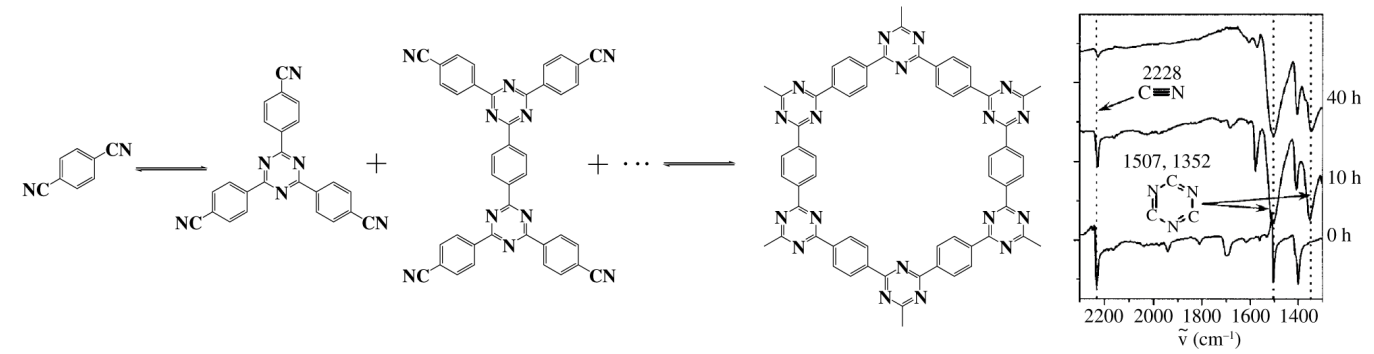

Figure 2: Trimerization of dicyanobenzene in molten $\mathrm{ZnCl}_{2}$ to trimers and oligomers and then to a covalent triazinebased framework (CTF-1) (Kuhn, Antonietti, and Thomas 2008), and FT-IR spectra following the progress of the condensation.

Moreover, Li et al. (2015) reported a novel benzimidazole-functionalized 2D COF material; they employed IR spectra to prove formation of the COF and irradiation stability. However, sometimes IR is not convincing enough; it often needs to cooperate with other analytical methods to explain the structure of COFs. For instance, Lei's group reported a three-dimensional (3D) graphene functionalized by COF thin film (Zha et al. 2015). The synthesis of $\mathrm{COF}_{\mathrm{DAAQ}-\mathrm{BTA}-3 \mathrm{DG}}$ is shown in Figure 3, and the appearance of the peaks of $\mathrm{N}-\mathrm{H}$ and $\mathrm{C}=\mathrm{O}$ (aldehyde) in the $\mathrm{COF}_{\mathrm{DAAQ}-\mathrm{BTA}-3 \mathrm{DG}}$ composite spectrum suggested the existence of unreacted monomers. In order to further determine the existence of some new chemical bonds, X-ray photoelectron spectroscopy was employed, which we would introduce in the following section.

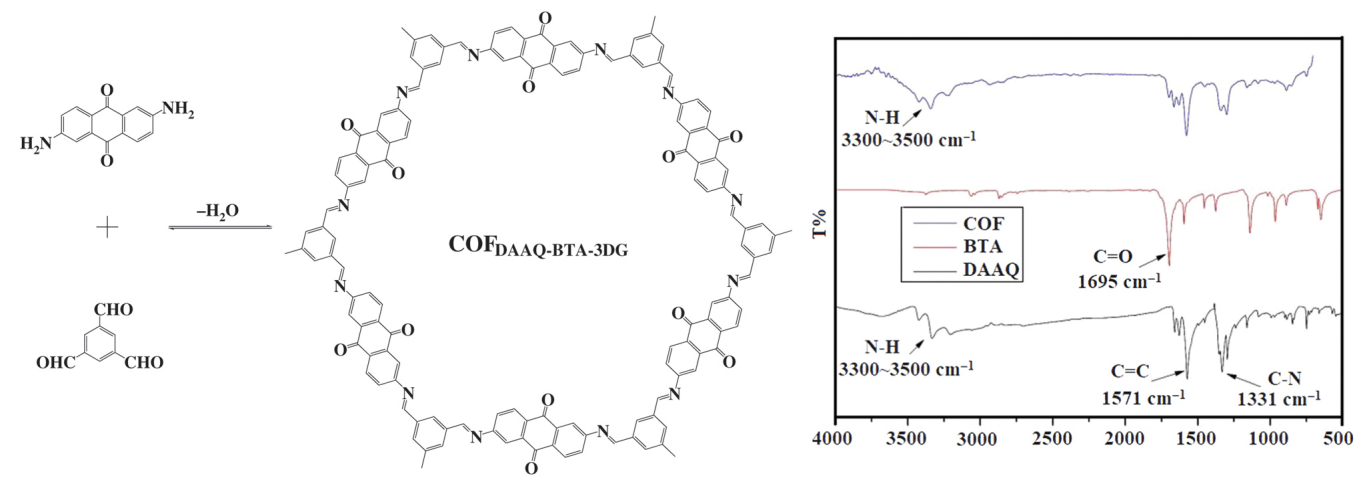

Figure 3: The synthesis and FT-IR spectra of $\mathrm{COF}_{\mathrm{DAAQ}-\mathrm{BTA}-3 \mathrm{DG}}$.

\subsection{X-ray photoelectron spectroscopy (XPS)}

XPS became an effective and common analytical method for determining the atom arrangement in nanoparticles and understanding the surface chemistry of materials (Bernardi et al. 2010, Sublemontier et al. 2014). Moreover, it can also be a powerful and available tool for characterizing the surface and bulk chemical structure of materials (Baer and Engelhard 2010 and Baer et al. 2010). As an important method of analysis and testing, XPS was also widely used in the analysis of COFs to determine the connection between the two monomers. As we introduced in the last section, Lei and co-workers reported a 2D COFs through the Schiff-base reaction of benzene-1,3,5-tricarbaldehyde with 2,6-diamino-anthraquinone moieties for modifying the 3D graphene (Zha et al. 2015). The XPS spectrum was used to support the conclusion of the IR spectrum. The figure in Zha et al. (2015) shows the presence of $\mathrm{N} 1 \mathrm{~s}$ at $400.22 \mathrm{eV}$ of DAAQ monomer, at 399.34 and $400.22 \mathrm{eV}$ of COF DAAQ-BTA-3DG composite, respectively. The peaks at 399.34 and $400.22 \mathrm{eV}$ were assigned to $\mathrm{N}$ in the imine bond and $\mathrm{NH}_{2}$ in the monomer, respectively. The peak area at $400.22 \mathrm{eV}$ is about one-third that of the one at $399.34 \mathrm{eV}$, which indicates that about one-fourth of the DAAQ monomer was unreacted. This is consistent with the results of IR analysis.

XPS not only can help us to make sure whether COF was synthesized, but also illustrate that the functional modification of COF is successful or not. Li et al. (2015) reported a benzimidazole-functionalized COF. The COF$\mathrm{COOH}$ was modified with previously synthesized 2-(2,4-dihydroxyphenyl)-benzimidazole (HBI), and a new solid-phase extractant (COF-HBI) with highly efficient sorption performance for uranium(VI) was consequently obtained. They employed FT-IR spectroscopy to characterize the product, but the IR spectrum did not provide all the information; thus they employed XPS for proving the expected product. FT-IR was first utilized for confirming whether the expected reaction (Figure 4) occurred, and then, XPS was used for further confirmation. 

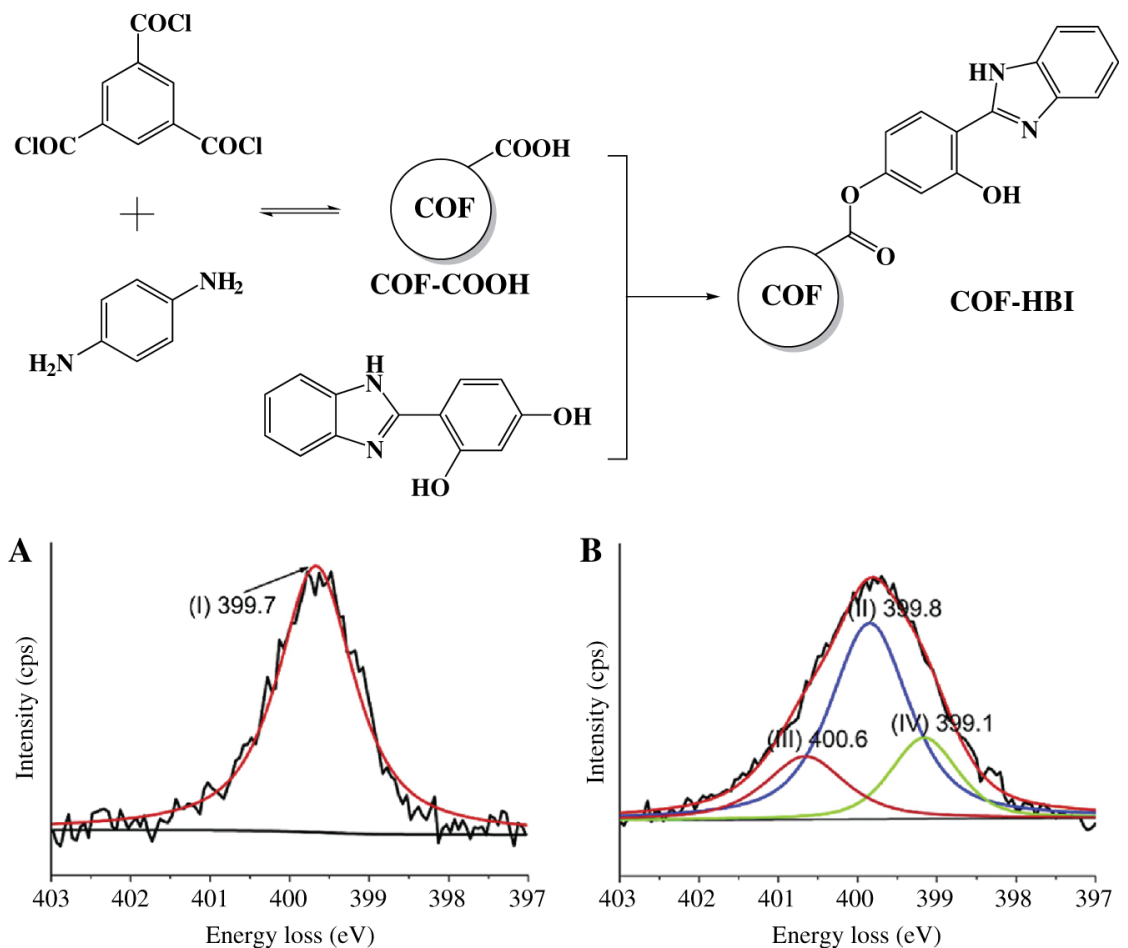

Figure 4: Preparations and N1s XPS spectra of COF-COOH and COF-HBI.

The figure in Li et al. (2015) showed the N 1s spectra of COF-COOH and COF-HBI. The single peak I at 399.8 $\mathrm{eV}$ corresponded to - NH- of amide in COF-COOH (Buch, Mohan, and Reddy 2008). Peak II at $399.8 \mathrm{eV}$ in the HBI-grafted sample was also assigned to $-\mathrm{NH}$ - of amide in this matrix. Peak III at $400.6 \mathrm{eV}$ and peak IV at 399.1 $\mathrm{eV}$ were attributed to $\mathrm{C}-\mathrm{N}$ and $\mathrm{C}-\mathrm{NH}-\mathrm{C}$ of the imidazole group, respectively (Charlier et al. 1997); thus the newly generated peaks (III and VI) could demonstrate that HBI was grafted to the COF matrix successfully as expected.

In addition, XPS was applied in more fields of COF too. For example, Li's group (Zhang et al. 2016b) ever reported a "stereoscopic" 2D super-microporous phosphazene-based COF in 2016, which could be used to adsorb uranium. Whether uranium is adsorbed or not can be ascertained by using XPS analysis. As XPS curves showed, the characterization peak of $\mathrm{U}_{4 \mathrm{f}}$ can be observed clearly by comparing the survey and the $\mathrm{U}_{4 \mathrm{f}}$ highresolution spectra of super-microporous phosphazene-based COF (MPCOF) and MPCOF-U, which indicated the sorption of uranium onto MPCOF, can indeed be approved.

\subsection{Nuclear magnetic resonance spectroscopy (NMR)}

In addition to IR and XPS, NMR is often used to analyze the structure of COFs. For example, CTF-0 was prepared by trimerization of 1,3,5-tricyanobenzene (Katekomol et al. 2013) by Thomas and co-workers, as shown in Figure 5 . 


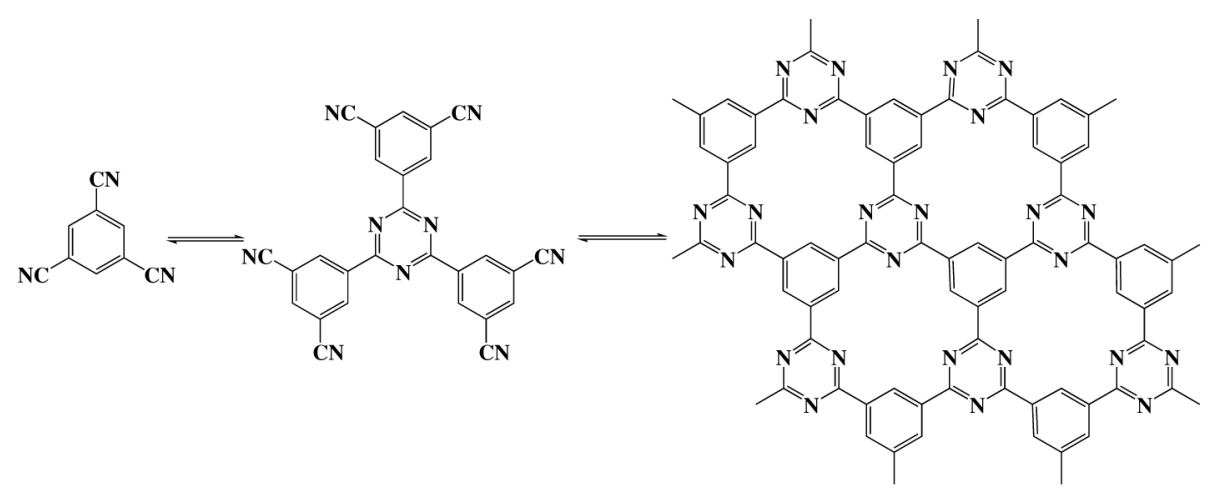

$\mathbf{A}$

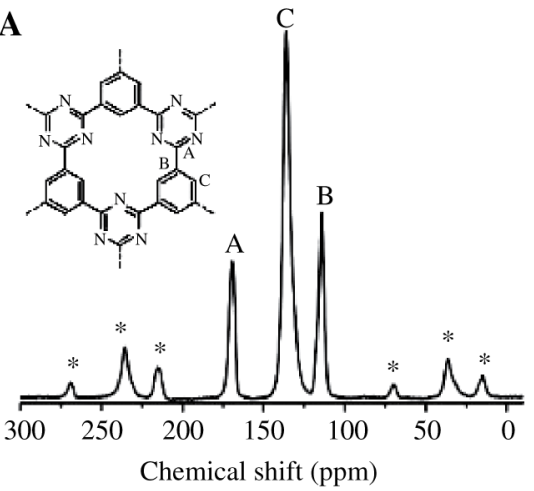

$\mathbf{B}$

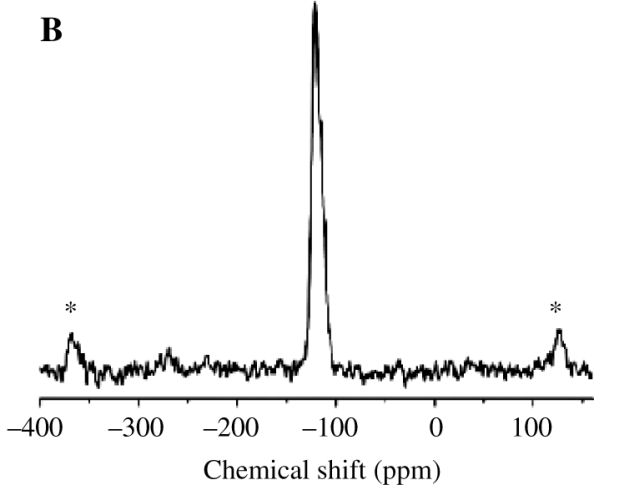

Figure 5: Trimerization of 1,3,5-tricyanobenzene.

(A) ${ }^{13} \mathrm{C}$ CP-MAS solid-state NMR and (B) 15N CP-MAS solid-state NMR of CTF-0.

The trimerization of 1,3,5-tricyanobenzene was confirmed by ${ }^{13} \mathrm{C}$ CP-MAS solid-state NMR. The peaks of three $\mathrm{sp}^{2}$ carbons of the CTF-0 were detected at $\delta=167,136$ and $114 \mathrm{ppm}$, respectively. Moreover, no peaks of residual cyano groups (110 ppm) were detected in the spectrum. The peak of triazine moiety detected at $\delta$ $=-121$ ppm by ${ }^{15} \mathrm{~N}$ solid-state NMR indicated that $\mathrm{N}$ was just present in the form of triazine rings and the product was expected (Katekomol et al. 2013).

More and more attention has been paid to tetrathiafulvalene (TTF) and its derivatives due to their $p$ electron-donating abilities and two reversible oxidation processes (Ding et al. 2014). The COF based on TTF was synthesized by Zhang's group in 2014 for the first time. The TTF-COF constructed by tetrathiafulvalenetetrabenzaldehyde and p-phenylenediamine (PPDA) with $-\mathrm{C}=\mathrm{N}-$ (Figure 6) provided potential for applications in electronic and optoelectronic materials. 


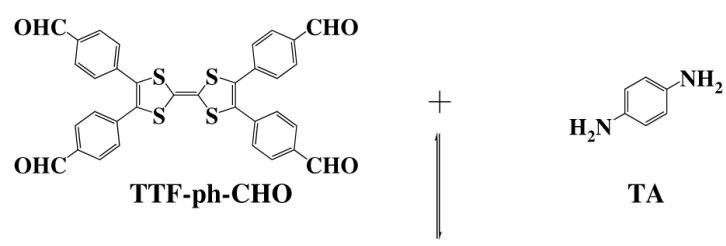

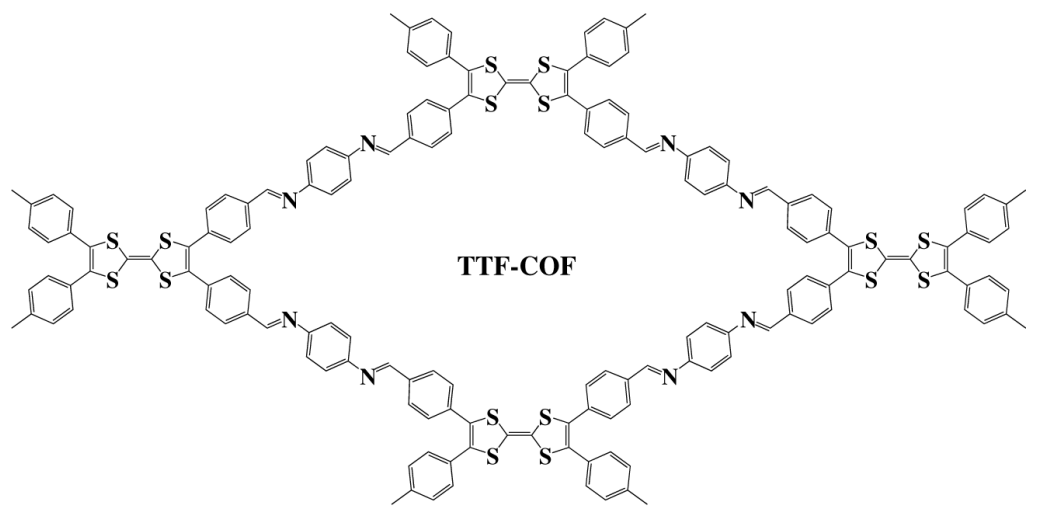

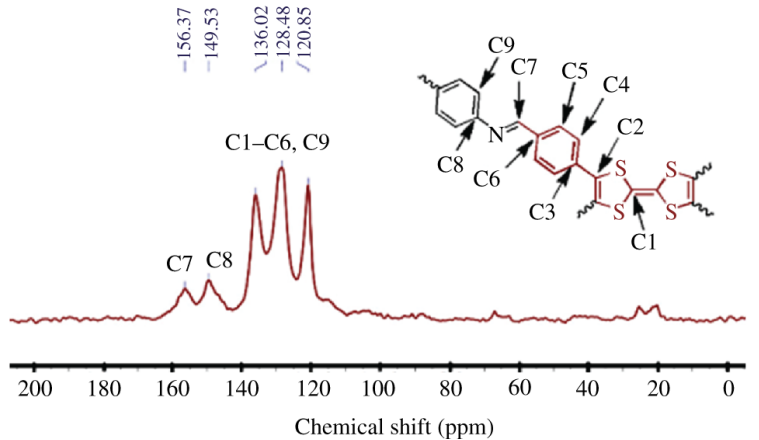

Figure 6: The synthesis ${ }^{13} \mathrm{C}$ and CP TOSS NMR spectrum of TTF-COF.

The resonance for the carbon of the $\mathrm{C}=\mathrm{N}$ bond at $157 \mathrm{ppm}$ was detected by ${ }^{13} \mathrm{C}$ cross-polarization with total suppression of sideband NMR spectroscopic methods (Ding et al. 2014), and the expected TTF-COF was determined.

\section{$2.4 \quad$ X-ray diffraction (XRD)}

The information about the crystal structure can be obtained by XRD. Sometimes the successful synthesis of the product can be judged by comparing the XRD pattern, and the stability of the product can be evaluated by observing the change in the XRD pattern.

A squaraine-linked mesoporous COF (CuP-SQ COF) was synthesized through the condensation of squaric acid with copper(II) 5,10,15,20-tetrakis(4-aminophenyl)porphyrin (TAP-CuP) (Figure 7) by Jiang's group (Nagai et al. 2013).
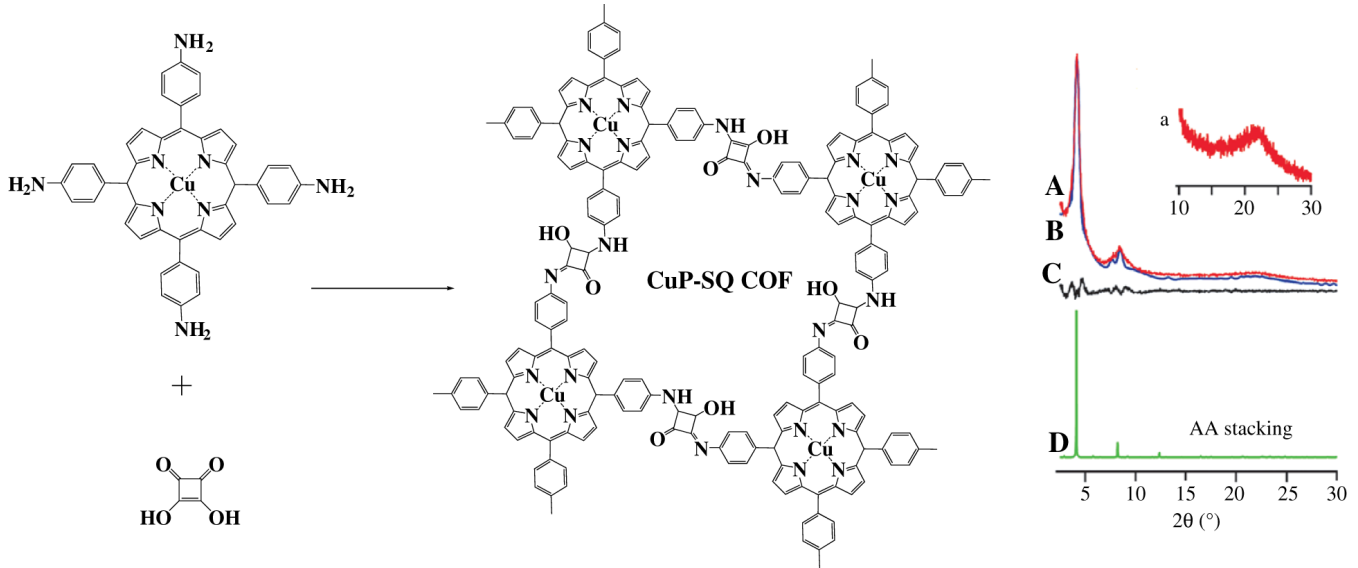
Figure 7: Synthesis of CuP-SQ COF and PXRD curves.

(A) Experimental CuP-SQ COF, (B) the Pawley-refined pattern, (C) the difference between two curves and (D) the simulated pattern with an AA stacking mode.

High crystallinity of CuP-SQ COFs was indicated by powder X-ray diffraction (PXRD) measurements. A strong peak at $4.168^{\circ}$ and relatively weak peaks at $8.56^{\circ}$ and $21.968^{\circ}$ could be observed from the experimental PXRD pattern; these peaks corresponded to 100, 200 and 001 facets, respectively (Nagai et al. 2013). The simulative PXRD pattern using the AA stacking structure agreed well with the experimental PXRD pattern. The results indicate that the 2D CuP-SQ COF consisted of AA-type stacks of the crystalline porphyrin zigzag-like sheets. The pore size was $2.1 \mathrm{~nm}$ measured by PXRD, which was identical to the value of the $\mathrm{N}_{2}$ adsorption and desorption experiment. Stability of the CuP-SQ COF was also evaluated by re-measuring PXRD patterns of the vacuum-dried sample after dispersed in different solvents for $24 \mathrm{~h}$. The PXRD patterns of the CuP-SQ $\mathrm{COF}$ in different solvents (methanol, benzene, and hexane, water and aqueous $\mathrm{HCl}$ solution) were the same as the original CuP-SQ COF, indicating that the COFs were stable in these common solvents (Nagai et al. 2013).

In addition to helping to understand the stability of the COF in different solvents, XRD can also be used to determine the stability of the COF after used repeatedly. COF-10 was discovered to present the highest capacity for ammonia (Doonan et al. 2010), prepared by condensation of 2,3,6,7,10,11-hexahydroxytriphenylene (HHTP) and 4,4'-biphenyldiboronic acid (Côté et al. 2007, Doonan et al. 2010) (Figure 8). XRD was utilized to test the stability of COF-10 after cycling of ammonia adsorption. The experimental results indicated that the structure of COF-10 was stable after use (Doonan et al. 2010).

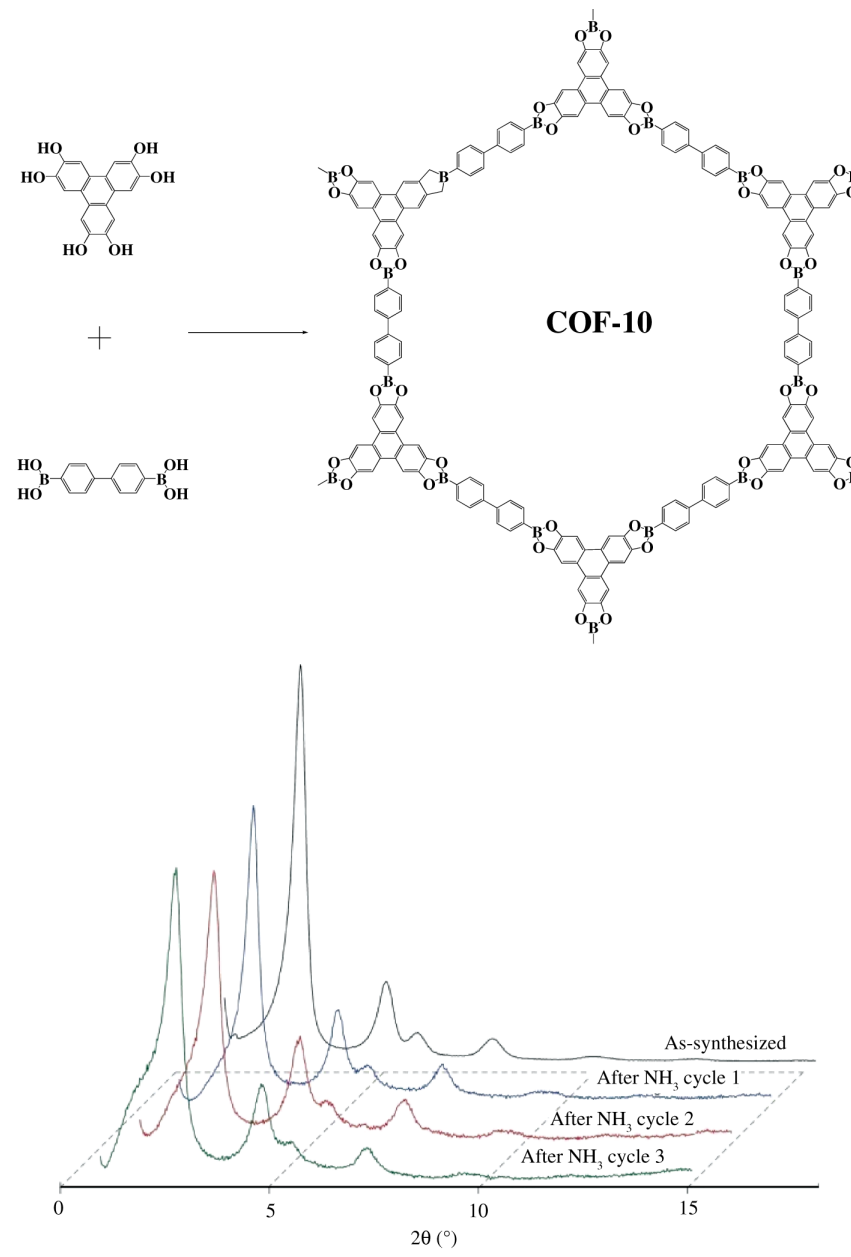

Figure 8: Synthesis process and XRD patterns after adsorbing $\mathrm{NH}_{3}$ for different cycles of COF-10.

The crystallinity of COFs is a distinctive feature as compared to other porous organic materials; single-crystal X-ray diffraction (SXRD) and rotating electron diffraction are also applied to the characterization of COFs. COF-320 was synthesized through the condensation of tetra-(4-anilyl)methane and 4,4'biphenyldialdehyde by Yaghi's group (Zhang et al. 2013). The crystal structure of COF-320 is determined by the rotation electron diffraction method for data collection. The suggested space group possibly is $\mathrm{I}_{1} \mathrm{md}$ or $\mathrm{I}_{2} \mathrm{~d}$. Yaghi and Wang's group developed a general procedure to grow large single crystals of 3D imine-based COFs (Ma et al. 2018). SXRD was employed to monitor the hydration of COF-300, and the molecular structure was characterized in detail. 


\subsection{Scanning electron microscope (SEM)}

SEM is an important and useful tool for observing and characterizing the surface structure and morphology of materials including COFs. Four crystalline 3D COFs (termed as COF-102, COF-103, COF-105 and COF-108) were reported by Yaghi's group in 2007 (El-Kaderi et al. 2007). In order to determine the phase purity and synthetic reproducibility of COF materials, SEM was exploited to scan all types of morphologies present in the samples. The SEM images of COF-102 and COF-103 revealed a spherical morphology; the surface of the particles was not smooth, but full of folds. The SEM image showed that COF-105 revealed platelet morphology with many granules, and the surface of particles was smooth. COF-108 revealed a deformed spherical morphology.

In 2012, two chemically stable (in acid and base) COF materials were synthesized by the Schiff base reactions (Kandambeth et al. 2012). The morphologies of these two materials were observed by SEM; besides, their stability was also studied through observing the transformation of morphology under different conditions. Each $\mathrm{COF}$ was submerged in water, $\mathrm{HCl}$ solutions and $\mathrm{NaOH}$ solutions, respectively, and then tested by SEM, XRD, FT-IR, and $\mathrm{N}_{2}$ adsorption-desorption experiments. The morphology of TpPa- 1 and TpPa- 2 under different conditions was demonstrated.

At present, COF formation based on strong dipole moments by a supramolecular strategy was reported (Salonen et al. 2016). A novel dione-COF was formed through the reaction between a new pyrene dione building block and HHTP, which presented excellent crystallinity and a large surface area. A loose and uniform internal structure can be observed, and the ordered porous structure was revealed by transmission electron microscope. Like SEM, TEM is also very important in the characterization of COFs, and it will be introduced in detail in the following section.

\subsection{Transmission electron microscope (TEM)}

TEM and corresponding analytical technology enrich people's understanding of the microstructure of the material. This method has been used for characterizing the dione-COF mentioned above. The typical porous structure of the dione-COF was presented clearly. The pores presented a hexagonal symmetry and the pore size was about $2.4 \pm 0.2 \mathrm{~nm}$ (Salonen et al. 2016). In 2015, a series of electron donor-acceptor COFs were constructed via direct polycondensation reactions by Jiang and co-workers (Jin et al. 2015). These materials have potential in charge separation and photoenergy conversions. The lattice and layer structure were clearly presented by high-resolution transmission electron microscope (HRTEM).

BDT-COF is a crystalline, porous and thermally stable material, formed by benzo[1,2-b:4,5-b']dithiophene2,6-diyldiboronic acid (BDTBA) and HHTP (Figure 9), and reported by Bein's group in 2014 (Medina et al. 2014). The lattice and layer structure can be observed by HRTEM. 


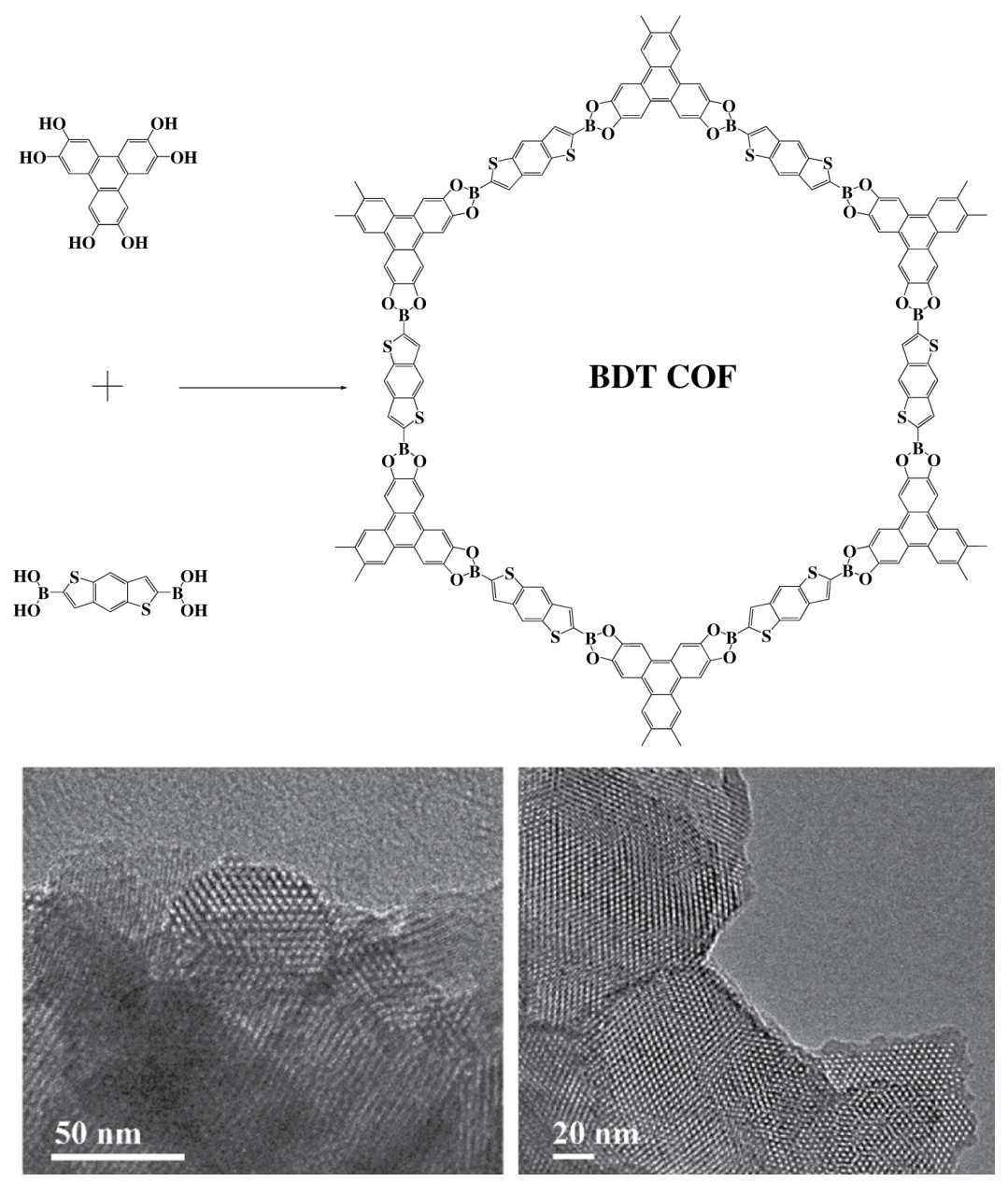

Figure 9: Synthesis reaction and TEM images of BDT-COF.

\subsection{Scanning tunneling microscope (STM)}

STh provide more information about the microscopic structure compared with TEM. For instance Wan and Wang's group reported COF formation from TTF with four benzaldehyde groups (4ATTF) and pphenylenediamine (PPDA) on the surface of highly oriented pyrolytic graphite (HOPG) (Dong et al. 2015), as shown in Figure 6.

The STM image showed that the condensation of 4ATTF and PPDA can form an ordered rhombus structure. The rhombus structure of molecules was present as bright spots in the STM image that can be attributed to electron-rich TTF cores. The measured lattice parameters of this rhombus structure are $a=b=2.5 \pm 0.2 \mathrm{~nm}$ and $\alpha=65^{\circ} \pm 2^{\circ}$.

Wan and Wang's group reported another surface covalent framework (SCOF) previously. A highly ordered SCOF was fabricated by a self-limiting solid-vapor interface reaction (Liu et al. 2013). SCOF-IC1 was obtained by condensation of 1,3,5-tris(4-aminophenyl)benzene on HOPG and gaseous terephthaldicarboxaldehyde in a closed system at $150^{\circ} \mathrm{C}$ for $3 \mathrm{~h}$. Similarly, SCOF-LZU1 was obtained by condensation of 1,3,5-triformyl-benzene and PPDA. The highly ordered honeycomb network structure was disclosed by STM, and the high-resolution STM images of SCOF-IC1 and SCOF-LZU1 were displayed, respectively. The lattice parameters of SCOF-IC1 and SCOF-LZU1 were measured to be $3.8 \pm 0.2$ and $2.2 \pm 0.2 \mathrm{~nm}$, which agreed well with the expected size of 3.92 and $2.25 \mathrm{~nm}$ by density functional theory (DFT) calculation, respectively.

The last example about STM was reported by De Feyter and co-workers in 2015 (Plas et al. 2015). COF1 was used as a template to stabilize the adsorption of fullerene $C_{60}$. These host-guest monolayers were easily patterned with STM lithography. The hexagonal grid structure of COF-1 can be observed through STM in Figure $10 \mathrm{~A}$. The pattern of bright blobs in the hexagonal grid indicated that each unit cell of COF- 1 contained one $\mathrm{C}_{60}$ molecule, as shown in Figure 10B. 

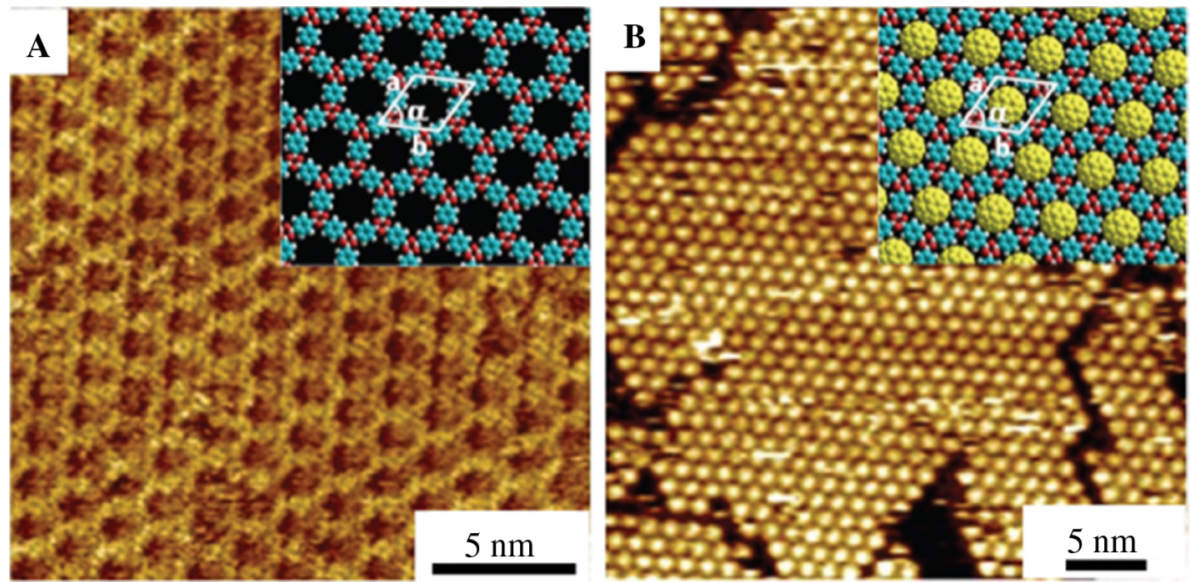

Figure 10: STM images of the COF-1 (A) and its host-guest co-assembly with $\mathrm{C}_{60}(\mathrm{~B})$.

The above examples showed that STM was very effective for observing the surface structure of COFs. In addition, there are other methods to research the SCOFs and COF composite, e.g. atomic force microscope.

\subsection{Atomic force microscope (AFM)}

A 3D graphene-functionalized COF, constructed with 2,6-diamino-anthraquinone and benzene-1,3,5tricarbaldehyde through the Schiff-base reaction (Zha et al. 2015), was used to prepare an electrode modified with good quality of conductivity. AFM morphological images showed the rough surface of COF DAAQ-BTA-3DG, and the thickness of the COF film was about $180 \mathrm{~nm}$.

Dichtel's group reported a patterned 2D phthalocyanine COF-oriented thin film grown selectively on singlelayer graphene (Colson et al. 2015). The patterned films were characterized by AFM; the results revealed that the thickness of the COF was $134 \pm 6 \mathrm{~nm}$, and an obvious contrast between COF-covered squares and surrounding wafers was observed in the phase image of the same region.

\subsection{Gas adsorption-desorption}

COF material is a kind of porous organic material with a small pore size and larger specific surface area. The pore properties of the COF, such as pore size, pore volume and specific surface area, can be measured by a nitrogen adsorption-desorption experiment. A novel 2D COF with two kinds of pore sizes (7.1 and $26.9 \AA)$ was synthesized by Chen's group (Zhou et al. 2014). The dual-pore COF was prepared via condensation of $4,4^{\prime}, 4^{\prime \prime}, 4^{\prime \prime \prime}$-(ethene-1,1,2,2-tetrayl)tetraaniline and terephthalaldehyde, as presented in Figure 11. 


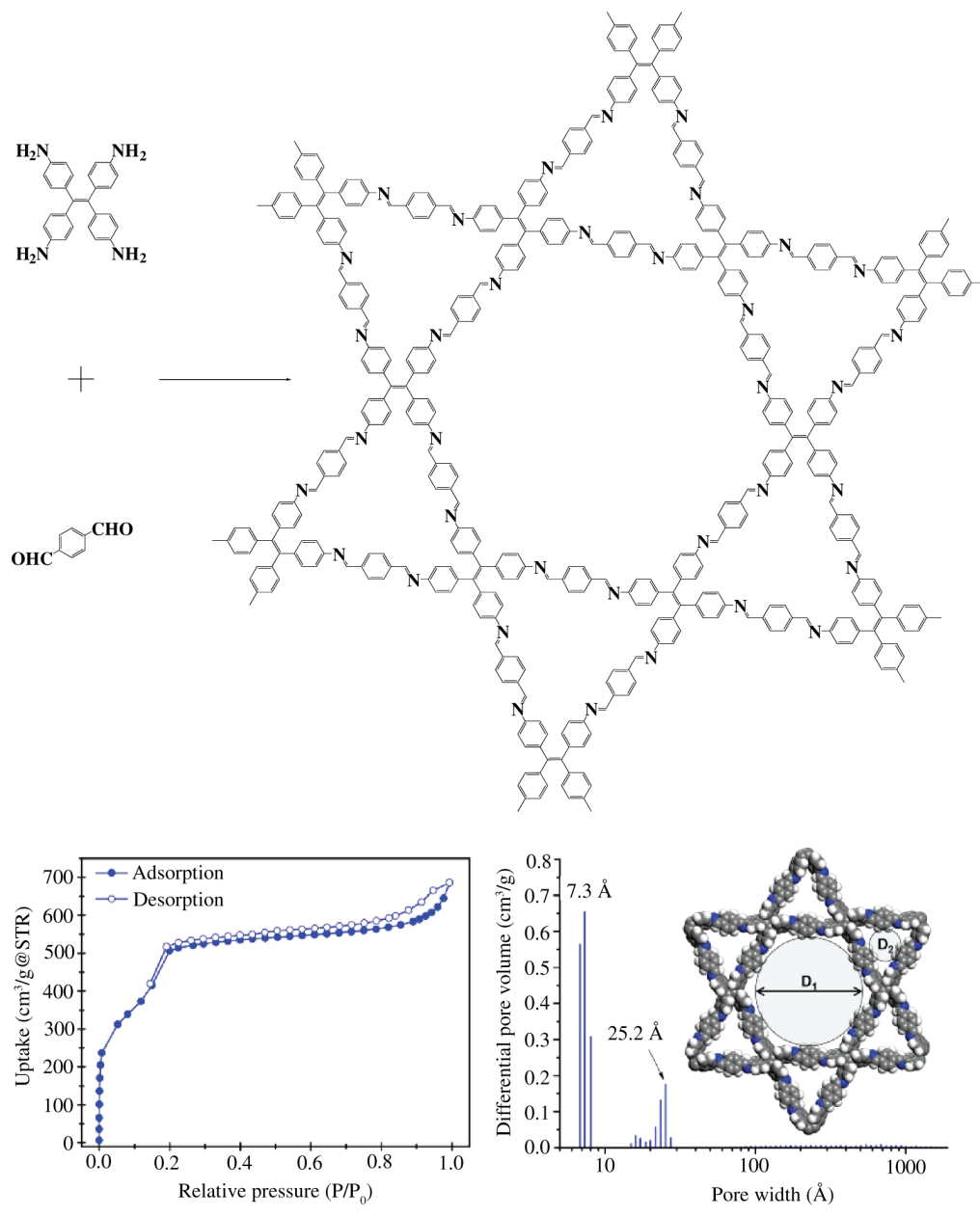

Figure 11: Synthesis and structure, $\mathrm{N}_{2}$ adsorption and desorption isotherm curves and pore size distribution profile of the dual-pore COF.

The formation of the dual-pore COF was proved by a $\mathrm{N}_{2}$ adsorption-desorption experiment. The $\mathrm{N}_{2}$ isotherm of the COF presented a sharp uptake below $P / P_{0}=0.01$ with a step between $P / P_{0}=0.15$ and 0.20 . The whole curve indicated that this COF possessed both microporous and mesoporous characters. The maximum $\mathrm{N}_{2}$ adsorption capacity was $686.1 \mathrm{~cm}^{3} \mathrm{~g}^{-1}$, the BET surface area was $1771.38 \mathrm{~m}^{2} \bullet \mathrm{g}^{-1}$ and the total pore volume of the COF was calculated to be $3.189 \mathrm{~cm}^{3} \mathrm{~g}^{-1}\left(P / P_{0}=0.99\right)$. Two pore size distributions at 7.3 and 25.2 $\AA$, respectively, were obtained by fitting the data with nonlocal DFT calculation, which were very close to the theoretical values of 7.1 and $26.9 \AA$ estimated by PM3 calculations.

The pore properties of the COF can influence its adsorption capacity significantly. The 3D COF-202 was synthesized by combining tert-butylsilane triol and tetra(4-dihydroxyboryl-henyl)methane in a mixed solution of dioxane and toluene in a sealed glass tube at $120^{\circ} \mathrm{C}$ for 3 days (Hunt et al. 2008). The porosity of COF-202 was characterized by an argon adsorption-desorption experiment at $87 \mathrm{~K}$ from 0 to 760 Torr. The gas adsorption and desorption curve indicated that COF-202 was a microporous material. Furthermore, the BET and Langmuir surface area values were 2690 and $3214 \mathrm{~m}^{2} \mathrm{~g}^{-1}$, respectively, and the pore volume was $1.09 \mathrm{~cm}^{3} \mathrm{~g}^{-1}$. Pore size distribution at $11 \AA$ was obtained by fitting the data with DFT calculations which fitted well with the expected pore size of 10 to $12 \AA$ based on the Cerius model (Hunt et al. 2008).

\section{The application of COFs in analytical chemistry}

The structure and properties of COFs can be clearly understood by means of a variety of analytical chemistry methods, such as IR, NMR, XPS, XRD, SEM, TEM, STM and so on. Due to outstanding merits, their applications in analytical chemistry have been reported too. At present, some fluorescent COFs have been applied in the detection of metal ions, explosives and small organic molecules. In addition, this novel material can also be applied to high-performance liquid chromatography technology. The applications of COF materials in analytical chemistry were introduced as follows. 


\subsection{Optical sensing application}

\subsubsection{Metalion}

Ding et al. (2016) reported a fluorescent COF for the detection of $\mathrm{Hg}^{2+}$. They synthesized the sulfoetherfunctional COF-LZU8 linked by hydrazine bonds under the solvent thermal condition (Figure 12). This material was stable in water and in common organic solvents such as dimethyl formamide (DMF), tetrahydrofuran (THF), dimethyl sulfoxide, chloroform and acetone. COF-LZU8 emitted fluorescence at 450 nm when excited at $390 \mathrm{~nm}$. The authors proved that the coordination effect of $\mathrm{Hg}^{2+}$ ions and sulfur elements resulted in fluorescence quenching. A total of $11.6 \%$ of the $\mathrm{Hg}^{2+}$ content resulted in $80 \%$ fluorescence quenching of COF-LZU8, which promised a sensitive and selective determination of $\mathrm{Hg}^{2+}$ by fluorescence quenching. It was very likely that $\mathrm{Hg}^{2+}$ first bound to the $S$ atoms and the electrons were then transferred from the $\pi$-conjugation framework to the unoccupied orbitals of $\mathrm{Hg}^{2+}$, resulting in the significant fluorescence quenching (Xue et al. 2017).

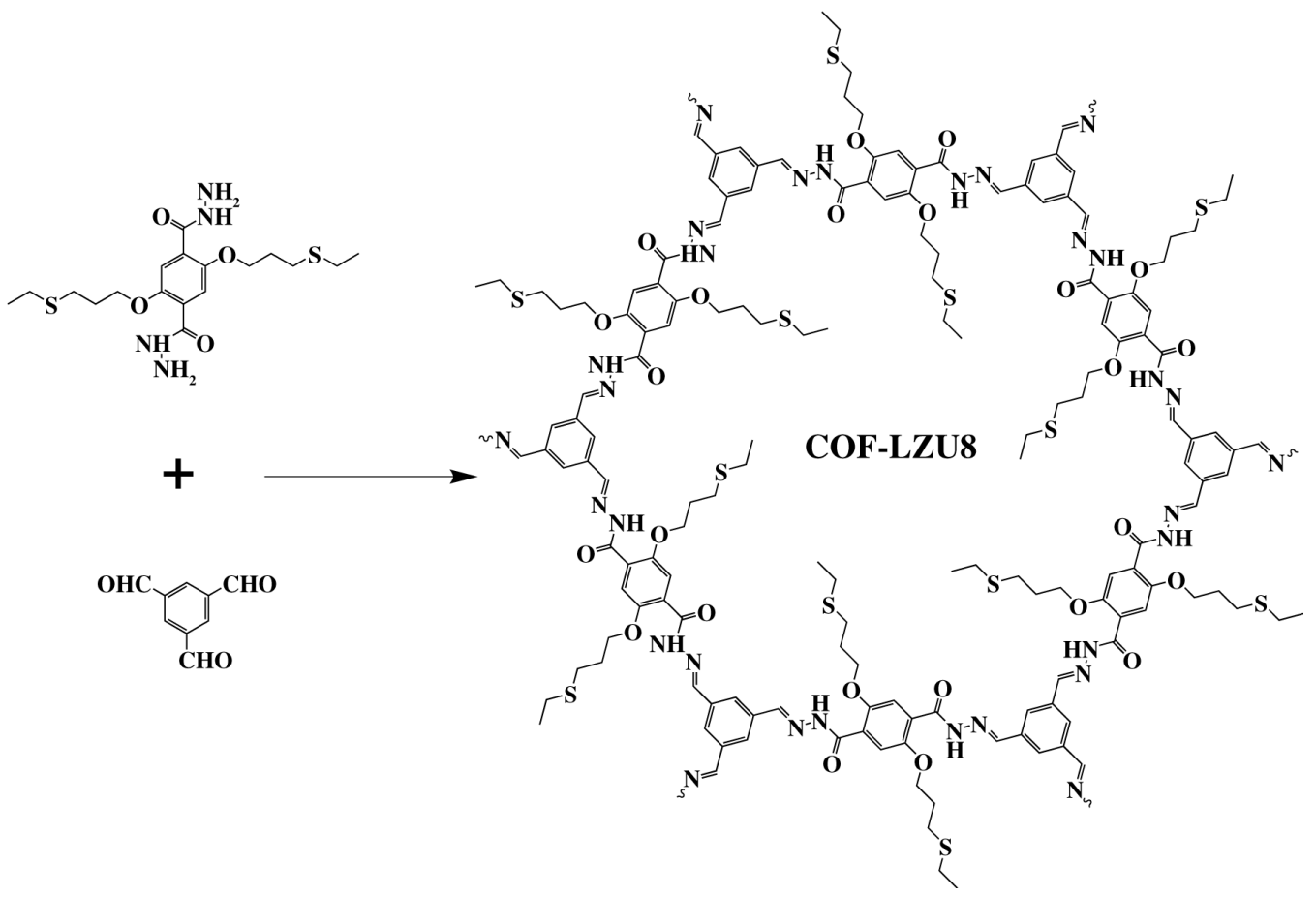

Figure 12: Synthesis and structure of COF-LZU8.

A hydrogen bond-assisted azine-linked COF (COF-JLU3) was synthesized by the reaction of 1,3,5-tris(3'tert-butyl-4'-hydroxy-5'-formylphenyl)benzene and hydrazine hydrate under solvothermal conditions (Figure 13) (Li et al. 2016). The experimental results indicated that COF-JLU3 had different fluorescence quenching for different concentrations of $\mathrm{Cu}^{2+}$ ions; the fluorescence emission intensity of COF-JLU3 was almost proportional to the concentration of $\mathrm{Cu}^{2+}$ ions in the range from 0 to $0.4 \mu \mathrm{M}$, and the detection limit of $\mathrm{Cu}^{2+}$ was $0.31 \mu \mathrm{M}$. The binding of $\mathrm{Cu}^{2+}$ ions led to an efficient quenching of the luminescence emission of the framework through a photo-induced electron transfer process from the COF to the metal ion. 


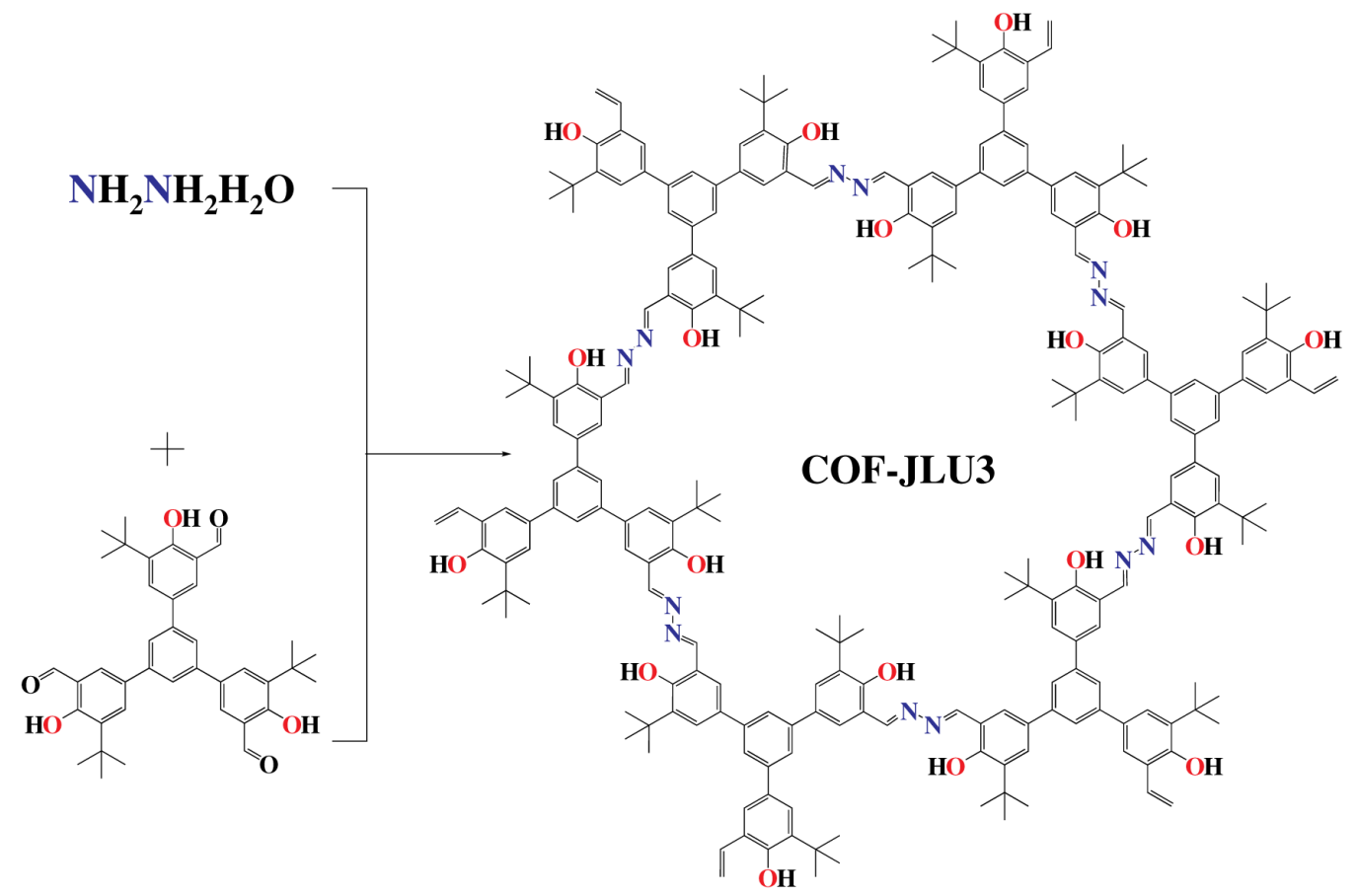

Figure 13: Synthesis and structure of COF-JLU3.

\subsubsection{Nitro-based explosives}

A rapid preparation of highly fluorescent nanoparticles of a new type of COF (SNW-1) was presented by Zhang et al. (2012), and this COF material exhibited extremely high sensitivity and selectivity, as well as fast response to nitroaromatic explosives such as 2,4,6-trinitrotoluene (TNT), 2,4,6-trinitrophenylmethyl-nitramine (Tetryl) and picric acid without interference by common organic solvent. SNW-1 obtained via polycondensation of terephthalaldehyde and melamine in dimethyl sulfoxide by a microwave-assisted method (Figure 14) showed a low detection limit (ppb level) and well precision for nitrobenzene (NB), 4-nitrotoluene, 2,6-dinitrotoluene (DNT), TNT and Tetryl by the fluorescence quenching experiments in the THF/ $\mathrm{H}_{2} \mathrm{O}(1: 9, \mathrm{v} / \mathrm{v})$ mixture. The strong quenching effect of nitro-aromatics on the fluorescence of the COF mainly resulted from dynamic quenching due to the transfer of the photoexcited electrons from the excited luminescence network donor to the electrondeficient nitro-aromatic acceptors and strong intermolecular interactions such as stacking effects between triazine rings in the polymer network and aromatic rings of nitroaromatic compounds (Xue et al. 2017).

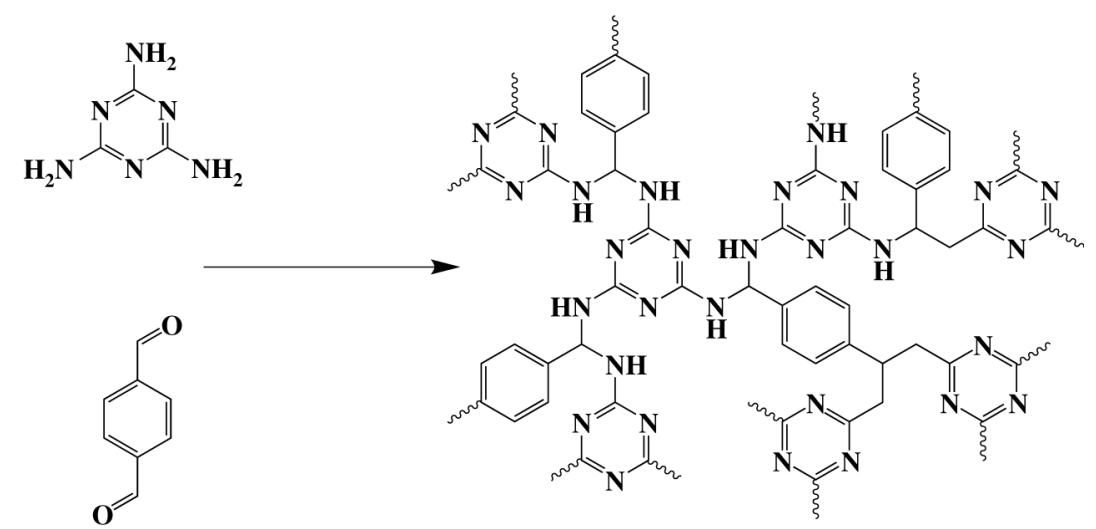

Figure 14: Synthesis and structure of SNW-1.

A 3D fluorescent COF was synthesized by Wang and co-workers in 2016 (Lin et al. 2016). This COF with new topology was synthesized by the condensation reaction of tetra(p-amino-phenyl)methane and 1,3,6,8-tetrakis(4formyl-phenyl) pyrene (Figure 15) and presented good fluorescence performance. The 3D-Py-COF was the first fluorescent 3D COF and could be used in explosive detection. 3D-Py-COF could emit strong yellow green luminescence at $484 \mathrm{~nm}$ under UV light irradiation at $408 \mathrm{~nm}$. PA could quench the fluorescence of 3D-Py-COF, and the quenching rate increased with the amount of PA in DMF. The quenching rate reached to $75 \%$ when 
the concentration of PA is $20 \mathrm{ppm}$. The quenching constant was estimated to be $3.1 \times 10^{4} \mathrm{M}^{-1}$ (Stern-Volmer model).

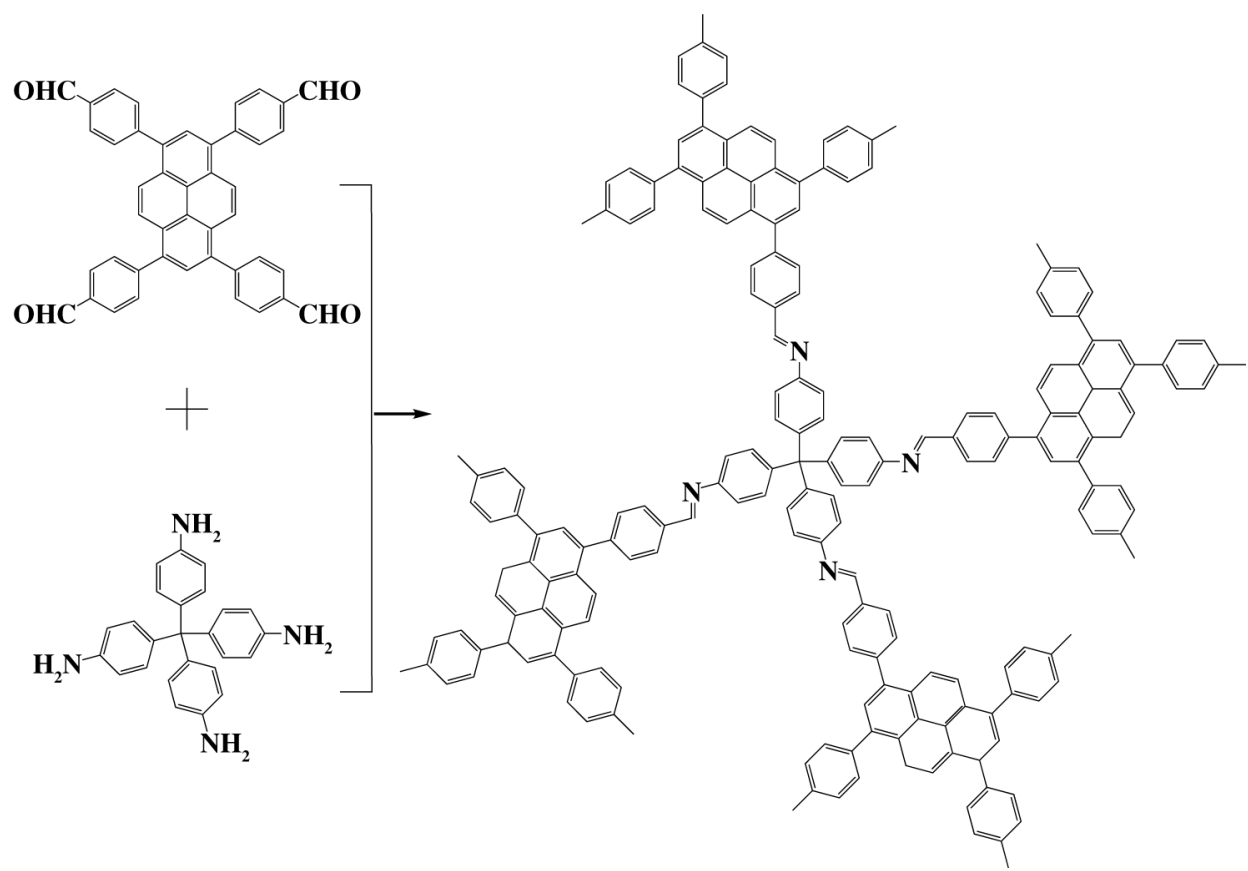

Figure 15: Synthesis and structure of 3D-Py-COF.

Another fluorescent COF, COF-LZU10, for detecting nitroaromatic compounds was reported by Ding (2014), which was constructed by condensation of a monomer containing thioether units and trimesurdehyde (Figure 16). This material emitted strong fluorescence under the irradiation of the ultraviolet lamp. The fluorescence quenching effects of COF-LZU10 were tested by some nitroaromatic compounds, such as DNT, TNT, NB, 4nitrophenol, PA, 4-nitrobenzoic acid, 1,4-benzoquinone, 1,3-dinitrobenzene and cyclotrimethylenetrinitramine; the result indicated that PA showed a significant quenching effect on luminescence of this material with high selectivity. The quenching process might obey a similar mechanism to the COF-LZU10, and the H-bonding interactions between the $-\mathrm{OH}$ units of $\mathrm{PA}$ and the open $\mathrm{N}$ atoms in the azine units on the pore walls played an important role in the fluorescence quenching. The fluorescence strength had a $75 \%$ decrease when $67 \mu \mathrm{mol}$ PA was added, and the detection limit was $4.7 \times 10^{-7} \mathrm{M}$.

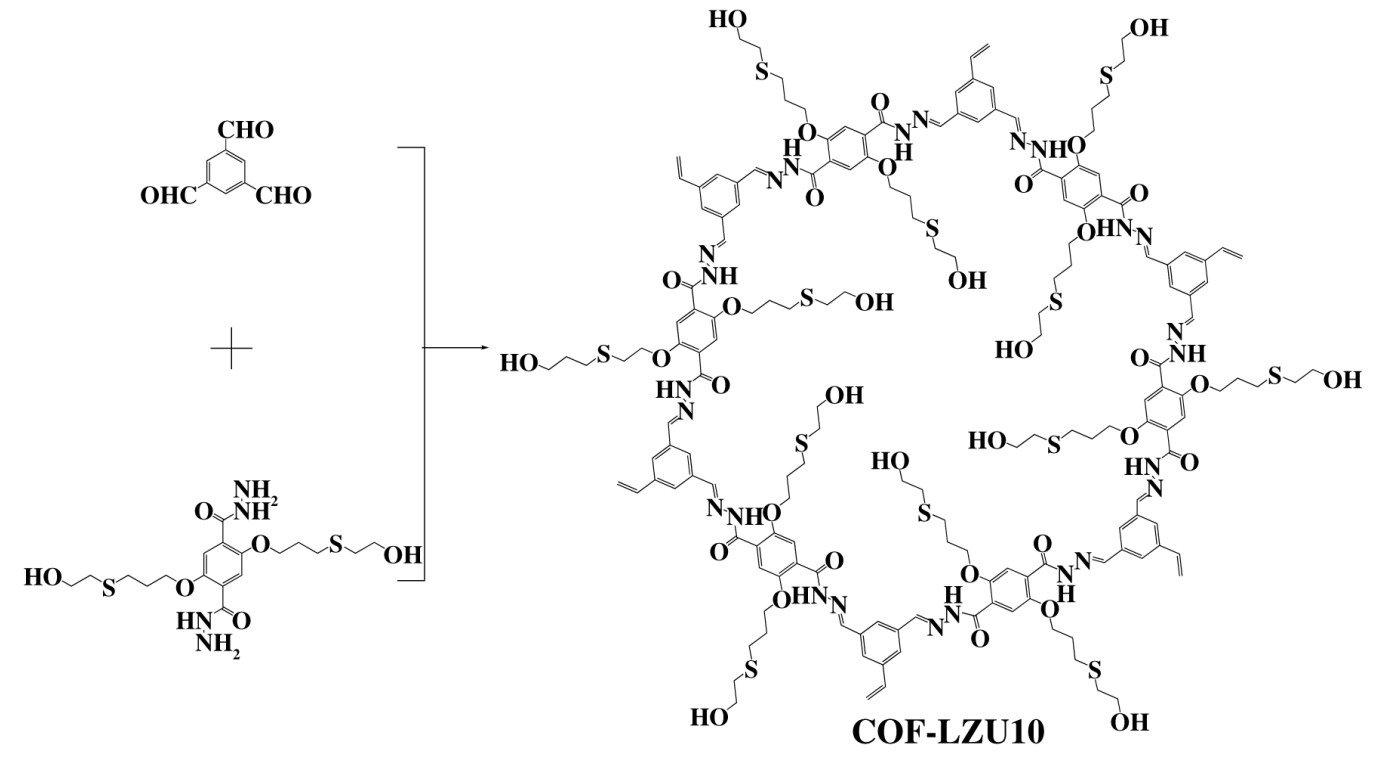

Figure 16: Synthesis and structure of COF-LZU10.

Jiang and co-workers reported a highly crystalline 2D COF based on the azine linkage in 2013 (Dalapati et al. 2013). The Py-azine COF was synthesized by the condensation reaction of 1,3,6,8-tetrakis(4formylphenyl)pyrene and hydrazine under solvothermal conditions (Figure 17). The Py-azine COF was chemically robust in various solvents and can be used for the detection of nitro-explosive too. The fluorescence of 
Py-azine COF solution was quenched quickly by PA vapor, and the fluorescence quenching degree reached 69\% when the concentration of PA was 70 ppm in acetonitrile. The hydroxy units of PA formed hydrogen-bonding interactions with the open nitrogen atoms in the azine units on the pore walls. The complexation of PA with the Py-azine COF formed a non-radiative complex that trapped the excitation energy of the COF skeletons, and the ground state complexation resulted in the static fluorescence quenching. Three nitro-groups resulted in the deficient $\pi$ system for driving the fluorescence quenching and strong interaction with the azine edges for promoting the quenching process. So, the Py-azine COF showed high sensitivity and selectivity to detection of PA.

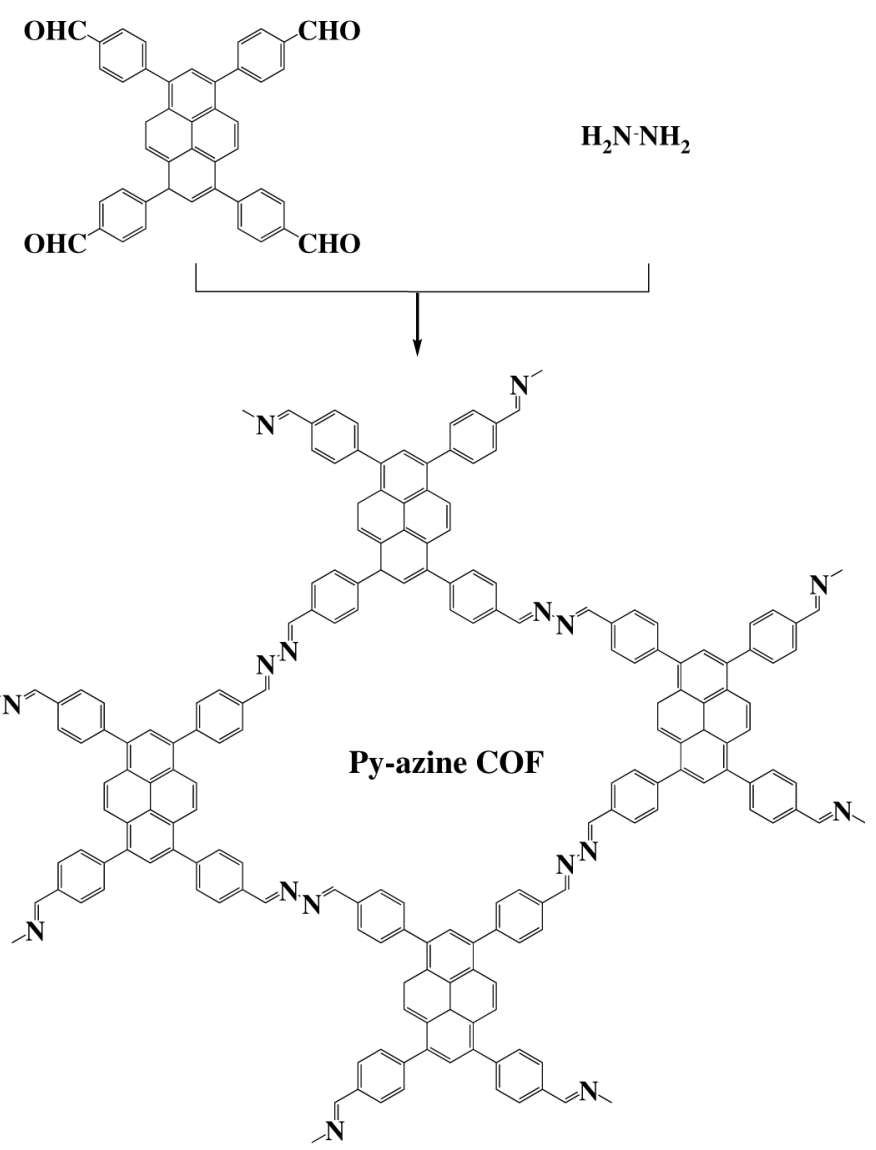

Figure 17: Synthesis and structure of Py-azine COF.

Recently, a new fluorescent polyimide covalent organic framework (PI-COF) has been successfully synthesized by condensation of TAP-CuP and perylenetracarboxylic dianhydride through the solvothermal method (Figure 18) by Xian and co-workers (Zhang et al. 2017a). The PI-COF has been successfully utilized as an efficient fluorescent probe for the highly sensitive and selective detection of PA. The PI-COF emitted the fluorescence at $500 \mathrm{~nm}$ under excitation at $375 \mathrm{~nm}$. The fluorescence intensity of the PI-COF was gradually reduced with the increasing concentration of PA $(0-21 \mu \mathrm{M})$. The result of the experiment exhibited a good linear response toward PA over the range from 0.5 to $10 \mu \mathrm{M}$ with a detection limit of $0.25 \mu \mathrm{M}$. The fluorescence was mainly attributed to the existence of a p-n heterojunction between TAP-CuP and 3,4,9,10-perylenetracarboxylic dianhydride units in the PI-COF. As an electron-deficient compound with nitro groups, PA could form a PI-CONs-picrate complex through the electron transfer between protonated PI-CONs and picrate anion $\left(\mathrm{PA}^{-}\right)$, so that the fluorescence of PI-CONs was quenched by PA. 


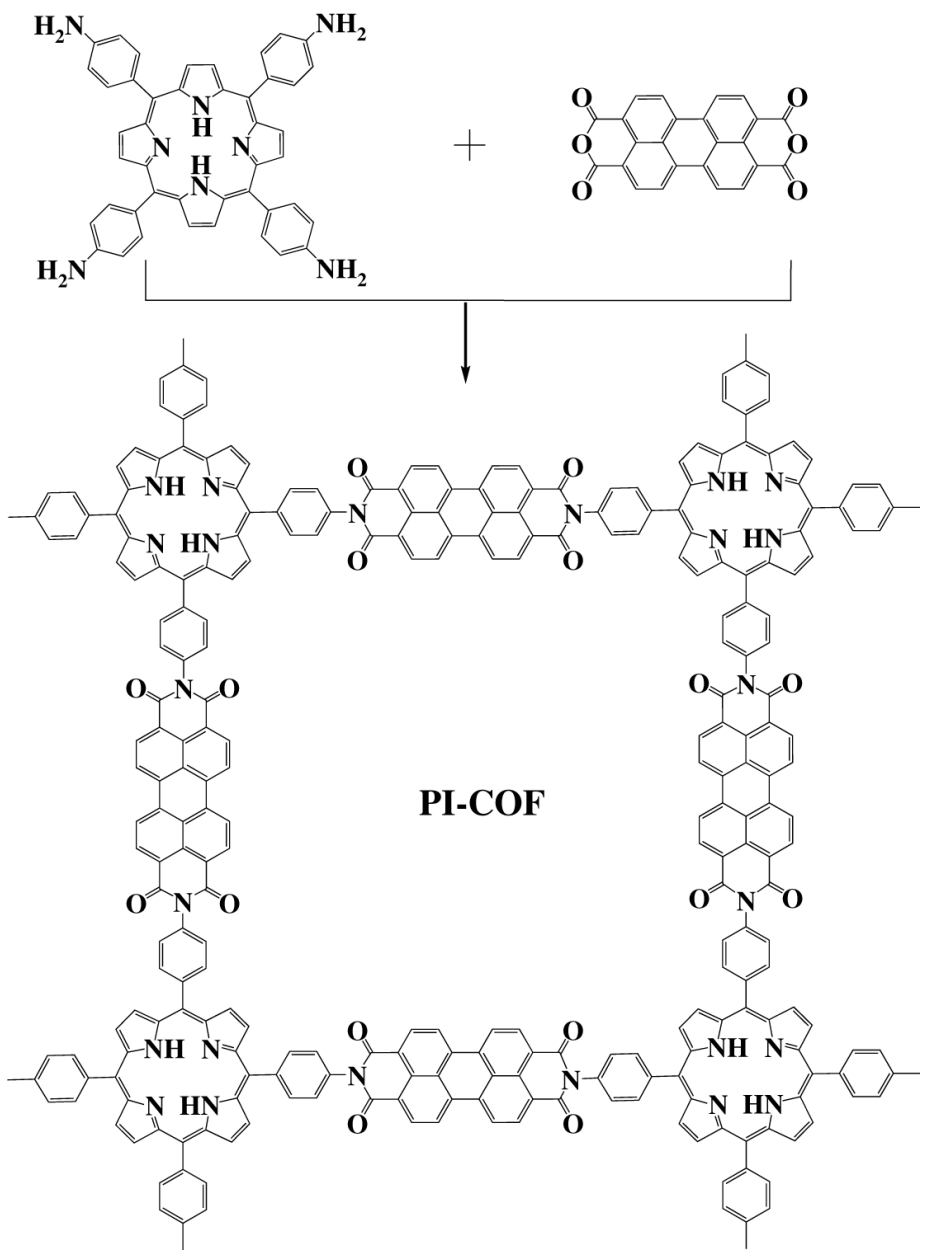

Figure 18: Synthesis and structure of PI-COF.

\subsubsection{Electron-rich arenes}

In addition to metal ions and nitroaromatic explosives, scientists have also developed some new COFs that can be used for detecting electron-rich arene vapors. Zheng and co-workers synthesized two new 2D COFs, TAT-COF-1 and TAT-COF-2, based on triazatruxene derivatives (Figure 19) (Xie et al. 2015). A total of 24 kinds of solvents were attempted to evaluate fluorescence influences, such as water, NB, methanol, 2-nitrotoluene, nbutanol, tetraethylene glycol, dioxane, N-methylpyrrolidone and so on. Experimental results showed that the fluorescence of TAT-COF-2 could be enhanced by electron-rich arenes (e.g. N-methylpyrrolidone, tetrahydrofuran, toluene, chlorobenzene), quenched by electron-deficient arenes (e.g. NB, methanol, 2-nitrotoluene, water, n-butanol, tetraethylene glycol, dioxane) and retained by ethanol, acetonitrile and acetone. The electron-rich nature of TAT-COFs may make it able to be used as an optoelectronic material on arene detecting. 


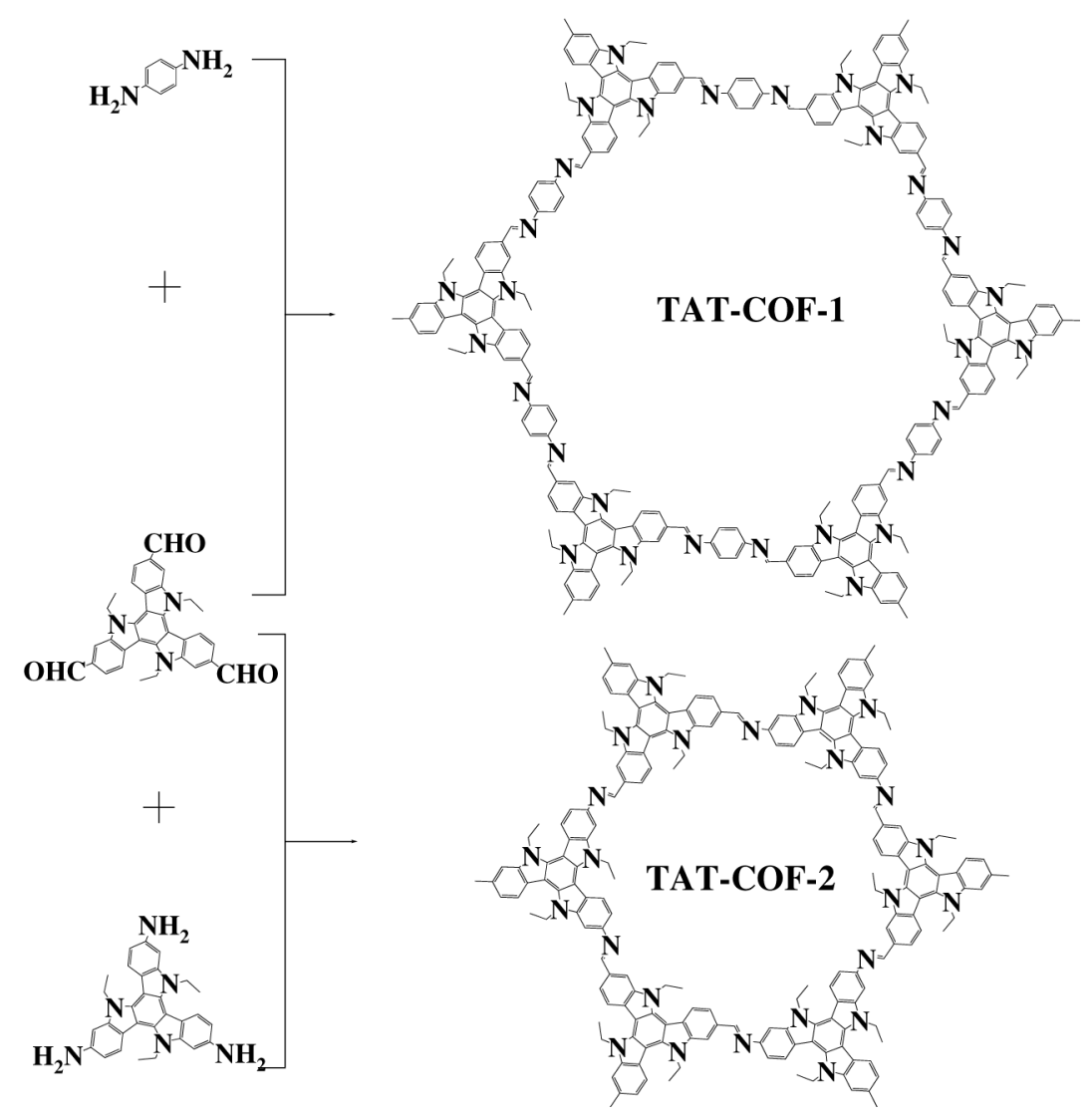

Figure 19: Synthesis and structure ofTAT-COF-1 and TAT-COF-2.

\subsection{4 $\mathrm{pH}$}

A $\beta$-ketoenamine-based covalent organic framework (COF-JLU4) was synthesized via condensation of 2,5dimethoxy terephthalohydrazide and triformyl phloroglucinol under solvothermal conditions (Figure 20) by Liu and co-workers in 2016 (Zhang et al. 2016a). This COF was the first COF-based fluorescent pH sensor in aqueous solutions and had good wettability for water. COF-JLU4 displayed an absorption band at $380 \mathrm{~nm}$ and an emission band at $568 \mathrm{~nm}$ in the solid state. The fluorescence intensity and wavelength of COF-JLU4 were strongly correlated with the $\mathrm{pH}$ value of the dispersed solution in the experimental $\mathrm{pH}$ range (0.9-13.0). The most acidic solution displayed the strongest fluorescence. When the $\mathrm{pH}$ changed from 9.0 to 13.0, the fluorescence intensity of COF-JLU4 decreased gradually. The weakest fluorescence was obtained at $\mathrm{pH}=13.0$. This research expanded the scope of COFs and constituted an important step toward porous crystalline organic architecture with designed functions. 


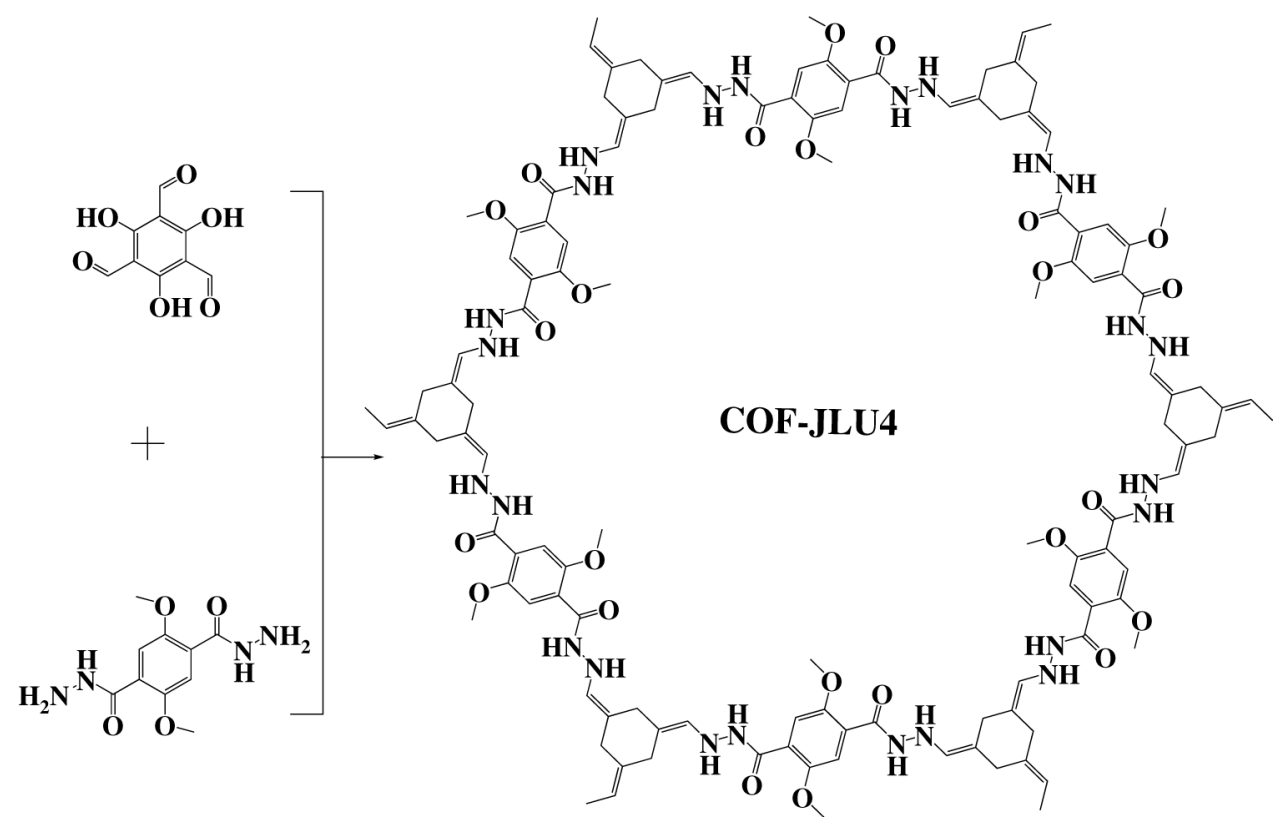

Figure 20: Synthesis and structure of COF-JLU4.

\subsubsection{Ammonia}

Jiang and co-workers (Dalapati et al. 2016) designed and synthesized a new highly emissive COF by introducing an aggregation-induced emission (AIE) mechanism. AIE-active units were integrated into the polygon vertices to yield a crystalline porous COF with periodic $\pi$-stacked columnar AIE arrays. These columnar AIE $\pi$-arrays dominated the luminescence of the COF, achieved an exceptional quantum yield via a synergistic structural locking effect of intralayer covalent bonding and interlayer noncovalent $\pi-\pi$ interactions and served as a highly sensitive sensor to ammonia down to the sub-ppm level. The tetraphenylethene-phenyl (TPE-Ph) COF was prepared by solvothermal condensation of 1,2,4,5-tetrahydroxybenzene and TPE-cored boronic acids in a mixture of dioxane and mesitylene (Figure 21). The TPE-Ph COF exhibited an absorption band at $390 \mathrm{~nm}$ and emitted a strong blue luminescence, with peak maxima at $500 \mathrm{~nm}$. Based on the Lewis acid-base interaction of boronate and ammonia, the TPE-Ph COF showed a highly sensitive and a rapid response to ammonia. 


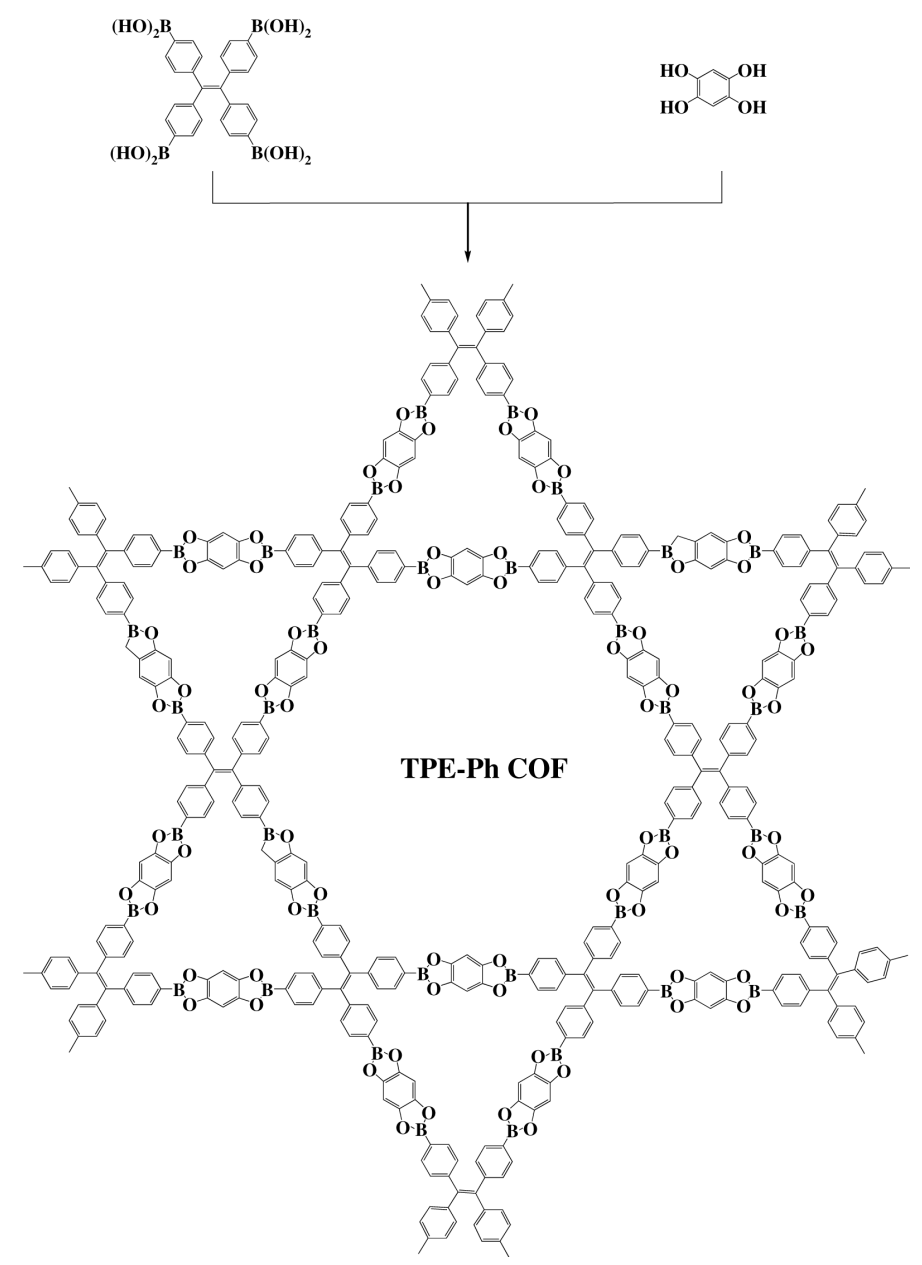

Figure 21: Synthesis and structure of TPE-Ph COF.

\subsubsection{Humidity}

Huang et al. (2013)) presented a rarely reported uniform COF nanofibers with a facile solvothermal route for the synthesis (Figure 22). The time-dependent growth of this crystalline COF nanofibers and morphology transformation process were observed, and were controlled by a novel dissolution-recrystallization growth mechanism enabled by the unique reversibility of $\mathrm{C}=\mathrm{N}$ bonds. This particular transformation process could be successfully applied to the fabrication of complex COF nanohybrid structures. The obtained COF/aramid nanohybrid material showed a humidity-responsive color-changing property. The fabric was light yellow in a dry atmosphere, but changed from light red to dark red gradually with increasing relative humidity from $20 \%$ to $100 \%$. 


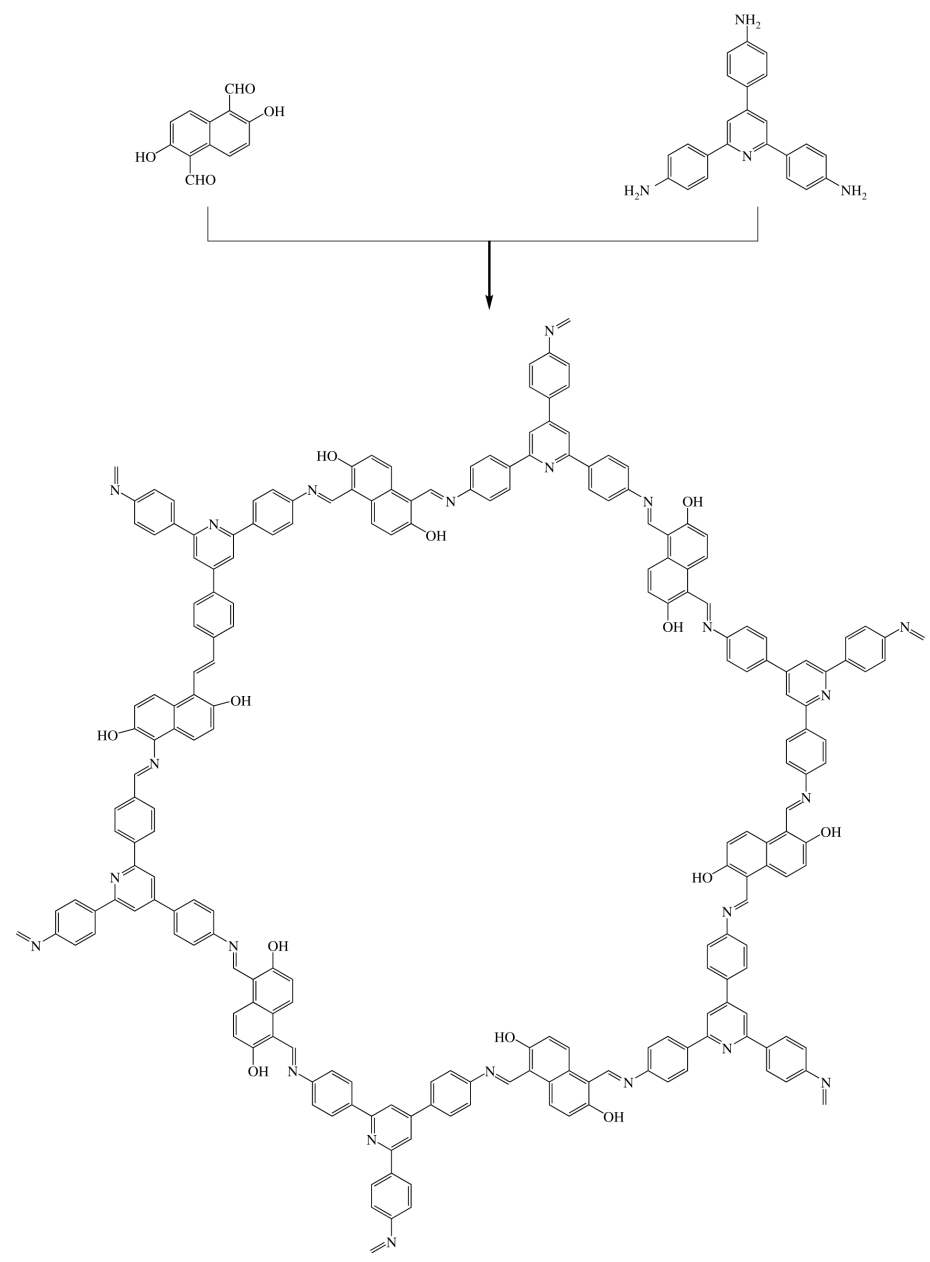

Figure 22: Synthesis of COFs by Condensation of 2,6-Dihydroxynaphthalene-1,5-dicarbaldehyde (DHNDA) and 2,4,6tris(4-aminophenyl)-pyridine (TAPP).

\subsection{Separation}

In addition to being used for analytical purpose, COFs can also be used as separation materials. However, related reported are less on separation application, which was focused mainly in solid-phase microextraction (SPME) and capillary electrophoresis.

Wang and co-workers synthesized a Schiff base network-1 (SNW-1) (Zhang et al. 2012) and used it as an SPME fiber-coating material (Wang et al. 2016b). The prepared fiber was exploited for SPME of phenols from honey samples. The developed method had large enrichment factors, low limits of detection, good linearity and repeatability for phenols. Chen and Chen (2017) prepared a novel COF-modified magnetic nanoparticle (M-COF-1) and employed this material to extract selectively paclitaxel in aqueous solutions, whose COF-1 was immobilized on the surface of magnetic nanoparticles by the bio-inspired polydopamine method through catechol groups. The modified particles exhibited good stability and excellent extraction efficiency to paclitaxel. By using M-COF-1 as selective sorbent, most of the matrix in plasma was removed and paclitaxel was selectively extracted. The first example of cross-linked hydrazone COF coating synthesized via thiol-ene click chemistry for SPME was reported by Wu et al. (2016). The modified fiber was applied in SPME of pesticide residues coupled with gas chromatography with an electron capture detector. A large enhancement factors, remarkable preconcentration ability and low detection limits were obtained under the optimum experimental conditions. As Zhang et al. (2017b) reported, COF-SCU1 was coated on the SPME fibers (prior functionalized stainless steel wires), applied to extract some volatile benzene homologues from indoor air samples. This method gave large enrichment factors, low limits of detection and good linearity for the determination of gaseous benzene homologues in indoor air samples.

The COF was used as the stationary phase in open-tubular capillary electrochromatography for the first time by Niu et al. (2016). COF-LZU1 was coated on the inner wall of the capillary as the stationary phase for separating organic molecules, because of the high surface area and aromaticity. The experimental data indicated excellent stability, high loading capacity and satisfactory reproducibility of the COF-LZU1-coated capillary. Bao 
et al. (2016) reported another example of OT-CEC modified by the COF. The obtained multilayer COF-5-coated capillary was used to separate neutral, acidic and basic analytes, and showed baseline separations as well as excellent stability.

In the end, COFs have attracted much attention because of their many advantages since the first report in 2005. It can be applied in the field of analytical chemistry, as described in the previous section. COFs can be constructed by monomers with specific properties due to the designability of structure and function. For instance, a fluorescent COF material can be constructed by monomers with a conjugated system, such as perylene, pyrene, HHTP, porphyrin, phthalocyanin and so on. In addition, a COF with high selectivity can be prepared by using specific functional groups. For example, a mercury fluorescence probe can be prepared by constructing with the sulfur-containing monomers. Finally, the determinand with different size can be detected selectively by COFs with different pore sizes, which was constructed by the monomers with different sizes. However, the construction of functional COFs with highly periodic and robust frameworks is still the challenge in the COF synthesis (Qian et al. 2017). Furthermore, the high cost and complexity of the synthesis process still restricts the development and application of COF materials. Though many challenges and difficulties still exist, the rapid development of COFs in recent years has predicted well for the bright future of this new type of porous organic materials. As the research moves along, more and more functional COFs will be synthesized and applied to the field of analytical chemistry.

\section{Conclusions}

As a kind of important porous organic crystal materials, COFs have shown great potential and research value in many spheres, for instance, gas storage and separation, photoelectric function fluorescence sensor, catalysis, drug delivery, dye and pollutant adsorption, electronic devices and so on. The structure and properties of the COF can be investigated and characterized through various methods, such as IR, XPS, NMR, XRD, SEM, TEM, STM, STM and gas adsorption-desorption experiment. These detection methods are just like "the eyes" that help us to "see" the crystal type, pores, layer structure and chemical bonds of COFs. COFs have also shown their extensive and important analytical applications along with going deep into of research gradually. Especially, some fluorescent COF materials have been applied in the detection of metal ions, explosives and small organic molecules and so on. In addition, they can be used for separation and enrichment of trace chemicals too. These trace substances in a solution can be clearly "seen" and captured by means of COFs. We believe that COFs can bring more and more surprise to us in analytical chemistry by the efforts of scientists, because of "chemistry = chem is try."

\section{Funding}

The authors are very grateful to National Natural Science Foundation of China, Funder Id: http://dx.doi.org/10.13039/501100001809 (Grant Number: 20873101, 21165024, 21765019), the Key Lab of Eco-Environments Related Polymer Materials of Ministry of Education, Key Lab of Polymer Materials of Gansu Province and Key Lab of Bioelectrochemistry and Environmental Analysis of Gansu Province for their supports.

\section{References}

Baer, D. R.; Engelhard, M. H. XPS analysis of nanostructured materials and biological surfaces. J. Electron Spectrosc. Relat. Phenom. 2010, 178$179,415-432$.

Baer, D. R.; Gaspar, D. J.; Nachimuthu, P.; Techane, S. D.; Castner, D. G. Application of surface chemical analysis tools for characterization of nanoparticles. Anal. Bioanal. Chem. 2010, 396, 983-1002.

Bai, L.; Phua, S. Z. F.; Lim, W. Q.; Jana, A.; Luo, Z.; Tham, H. P.; Zhao, L.; Gao, Q.; Zhao, Y. Nanoscale covalent organic frameworks as smart carriers for drug delivery. Chem. Commun. 2016, 52, 4128-4131.

Bao, T.; Tang, P.; Kong, D.; Mao, Z.; Chen, Z. Polydopamine-supported immobilization of covalent-organic framework-5 in capillary as stationary phase for electrochromatographic separation. J. Chromatogr. A 2016, 1445, 140-148.

Ben, T.; Ren, H.; Ma, S.; Cao, D.; Lan, J.; Jing, X.; Wang, W.; Xu, J.; Deng, F.; Simmons, J.; Qiu, S.; Zhu, G. Targeted synthesis of a porous aromatic framework with high stability and exceptionally high surface area. Angewandte Chemie International Edition 2009, 48, 9457-9460.

Bernardi, F.; Fecher, G. H.; Alves, M. C. M.; Morais, J. Unraveling the formation of core-shell structures in nanoparticles by S-XPS. J. Phys. Chem. Lett. 2010, 1, 912-917. 
Buch, P. R.; Mohan, D. J.; Reddy, A.V. R. Preparation, characterization and chlorine stability of aromatic-cycloaliphatic polyamide thin film composite membranes. J. Membr. Sci. 2008, 309, 36-44.

Budd, P. M.; Chanem, B. S.; Makhseed, S.; McKeown, N. B.; Msayib, K. J.; Tattershall, C. E. Polymers of intrinsic microporosity (PIMs): robust, solution-processable, organic nanoporous materials. Chem. Commun. 2004, 2, 230-231.

Charlier, J.; Detalle, V.; Valin, F.; Bureau, C.; Lécayon, G. Study of ultrathin polyamide-6,6 films on clean copper and platinum. J. Vac. Sci. Technol. A 1997, 15, 353-364.

Chen, Y.; Chen, Z. In situ fabrication of label-free optical sensing paper strips for the rapid surface-enhanced Raman scattering (SERS) detection of brassinosteroids in plant tissues. Talanta 2017, 165, 188-193.

Chen, L.; Furukawa, K.; Gao, J.; Nagai, A.; Nakamura, T.; Dong, Y.; Jiang, D. Photoelectric covalent organic frameworks: converting open lattices into ordered donor-acceptor heterojunctions. J. Am. Chem. Soc. 2014, 136, 9806-9809.

Colson, J. W.; Mann, J. A.; DeBlase, C. R.; Dichtel, W. R. Patterned growth of oriented 2D covalent organic framework thin films on single-layer graphene. J. Polym. Sci. Polym. Chem. 2015, 53, 378-384.

Cooper, A. I. Conjugated microporous polymers. Adv. Mater. 2009, 21, 1291-1295.

Corma, A. From microporous to mesoporous molecular sieve materials and their use in catalysis. Chem. Rev. 1997, 97, $2373-2420$.

Côté, A. P.; Benin, A. I.; Ockwig, N. W.; O'Keeffe, M.; Matzger, A. J.; Yaghi, O. M. Porous, crystalline, covalent organic frameworks. Science $\mathbf{2 0 0 5}$, 310, 1166-1170.

Côté, A. P.; El-Kaderi, H. M.; Furukawa, H.; Hunt, J. R.; Yaghi, O. M. Reticular synthesis of microporous and mesoporous 2D covalent organic frameworks. J. Am. Chem. Soc. 2007, 129, 12914-12915.

Dalapati, S.; Jin, S. B.; Gao, J.; Xu, Y. H.; Nagai, A.; Jiang, D. An Azine-linked covalent organic framework. J. Am. Chem. Soc. 2013, 135, 1731017313

Dalapati, S.; Jin, E.; Addicoat, M.; Heine, T.; Jiang, D. Highly emissive covalent organic frameworks. J. Am. Chem. Soc. 2016, 138, 5797-5800.

Davis, M. E.; Lobo, R. F. Zeolite and molecular sieve synthesis. Chem. Mater. 1992, 4, 756-768.

Ding, S. Y. Functional Covalent Organic Frameworks: Designed Synthesis, Characterization, and Application, PhD thesis.Lanzhou University, Lanzhou, 2014.

Ding, S. Y.; Wang, W. Covalent organic frameworks (COFs): from design to applications. Substrate orientation effect in the on-surface synthesis of tetrathiafulvalene-integrated single-layer covalent organic frameworks. Chem. Soc. Rev. 2013, 42, 548-568.

Ding, H.; Li, Y.; Hu, H.; Sun, Y.; Wang, J.; Wang, C.; Wang, C.; Zhang, G.; Wang, B.; Xu, W.; Zhang, D. A tetrathiafulvalene-based electroactive covalent organic framework. Chem. Eur. J. 2014, 20, 14614-14618.

Ding, S. Y.; Cao, J.; Wang, Q.; Zhang, Y.; Song, W. G.; Su, C. Y.; Wang, W. Chemoenzymatic synthesis of cryptophycin anticancer agents by an ester bond-forming non-ribosomal peptide synthetase module. J. Am. Chem. Soc. 2011a, 133, 19816-19822.

Ding, X.; Chen, L.; Honsho, Y.; Feng, X.; Saengsawang, O.; Guo, J.; Saeki, A.; Seki, S.; Irle, S.; Nagase, S.; Parasuk, V.; Jiang, D. Formation of SnO2 hollow nanospheres inside mesoporous silica nanoreactors. J. Am. Chem. Soc. 2011b, 133, 14510-14513.

Ding, X.; Guo, J.; Feng, X.; Honsho, Y.; Cuo, J.; Seki, S.; Maitarad, P.; Saeki, A.; Nagase, S.; Jiang, D. Synthesis of metallophthalocyanine covalent organic frameworks that exhibit high carrier mobility and photoconductivity. Angew. Chem. Int. Ed. 2011c, 50, 1289-1293.

Ding, S. Y.; Dong, M.; Wang, Y. W.; Chen, Y. T.; Wang, H. Z.; Su, C. Y.; Wang, W. Thioether-based fluorescent covalent organic framework for selective detection and facile removal of mercury(II). J. Am. Chem. Soc. 2016, 138, 3031-3037.

Dong, W.; Wang, L.; Ding, H.; Zhao, L.; Wang, D.; Wang, C.; Jun, L. Exceptional ammonia uptake by a covalent organic framework. Langmuir 2015, 31, 11755-11759.

Doonan, C. J.; Tranchemontagne, D. J.; Clover, T. G.; Hunt, J. R.; Yaghi, O. M. Exceptional ammonia uptake by a covalent organic framework. Nat. Chem. 2010, 2, 235-238.

El-Kaderi, H. M.; Hunt, J. R.; Mendoza-Cortés, J. L.; Côté, A. P.; Taylor, R. E.; O’Keeffe, M.; Yaghi, O. M. Designed synthesis of 3D covalent organic frameworks. Science 2007, 316, 268-272.

Fang, Q.; Gu, S.; Zheng, ].; Zhuang, Z.; Qiu, S.; Yan, Y. 3D microporous base-functionalized covalent organic frameworks for size-selective catalysis. Angew. Chem. Int. Ed. 2014, 53, 2878-2882.

Fang, Q.; Wang, J.; Gu, S.; Kaspar, R. B.; Zhuang, Z.; Zheng, J.; Guo, H.; Qiu, S.; Yan, Y. 3D porous crystalline polyimide covalent organic frameworks for drug delivery. J. Am. Chem. Soc. 2015, 137, 8352-8355.

Feng, X.; Liu, L.; Honsho, Y.; Saeki, A.; Seki, S.; Irle, S.; Dong, Y.; Nagai, A.; Jiang, D. High-rate charge-carrier transport in porphyrin covalent organic frameworks: switching from hole to electron to ambipolar conduction. Angew. Chem. Int. Ed. 2012, 51, 2618-262.

Graetz, J. New approaches to hydrogen storage. Chem. Soc. Rev. 2009, 38, 73-82.

Guo, L.; Zeng, X.; Cao, D. Porous covalent organic polymers as luminescent probes for highly selective sensing of Fe3+ and chloroform: functional group effects. Sens. Actuators B: Chem. 2016, 226, 273-278.

Harbuzaru, B. V.; Corma, A.; Rey, F.; Atienzar, P.; Jord, J. L.; García, H.; Ananias, D.; Carlos, L. D.; Rocha, J. Metal-organic nanoporous structures with anisotropic photoluminescence and magnetic properties and their use as sensors. Angew. Chem. Int. Ed. 2008, 120, 1096-1099.

Huang, W.; Jiang, Y.; Li, X.; Li, X.; Wang, J.; Wu, Q.; Liu, X. Solvothermal synthesis of microporous, crystalline covalent organic framework nanofibers and their colorimetric nanohybrid structures. ACS Appl. Mater. Interfaces 2013, 5, 8845-8849.

Hunt, J. R.; Doonan, C. J.; LeVangie, J. D.; Côté, A. P.; Yaghi, O. M. Reticular synthesis of covalent organic borosilicate frameworks. J. Am. Chem. Soc. 2008, 130, 11872-11873.

Jiang, J. X.; Su, R.; Trewin, A.; Wood, C. D.; Campbell, N. L.; Niu, H.; Dickinson, C.; Canin, A. Y.; Rosseinsky, M. J; Khimyak, Y. Z.; Cooper, A. I. Conjugated microporous poly(aryleneethynylene) networks. Angew. Chem. Int. Ed. 2007, 46, 8574-8578.

Jin, S.; Supur, M.; Addicoat, M.; Furukawa, K.; Chen, L.; Nakamura, T.; Fukuzumi, S.; Irle, S.; Jiang, D. Creation of superheterojunction polymers via direct polycondensation: segregated and bicontinuous donor-acceptor p-columnar arrays in covalent organic frameworks for longlived charge separation. J. Am. Chem. Soc. 2015, 137, 7817-7827.

Kandambeth, S.; Mallick, A.; Lukose, B.; Mane, M. V.; Heine, T.; Banerjee, R. Construction of crystalline 2D covalent organic frameworks with remarkable chemical (acid/base) stability via a combined reversible and irreversible route. J. Am. Chem. Soc. 2012, 134, 19524-19527. 
Katekomol, P.; Roeser, ].; Bojdys, M.; Weber, ].; Thomas, A. Covalent triazine frameworks prepared from 1,3,5-tricyanobenzene. Chem. Mater. 2013, 25, 1542-1548.

Kitagawa, S.; Kitaura, R.; Noro, S. Functional porous coordination polymers. Angew. Chem. Int. Ed. 2004, 43, 2334-2375.

Kuhn, P.; Antonietti, M.; Thomas, A. Porous, Covalent triazine-based frameworks prepared by ionothermal synthesis. Angew. Chem. Int. Ed. 2008, 47, 3450-3453.

Kumar, P.; Deep, A.; Kim, K. H. Metal organic frameworks for sensing applications. Trends Anal. Chem. 2015, 73, 39-53.

Li, H.; Eddaoudi, M.; Groy, T. L.; Yaghi, O. M. Establishing microporosity in open metal-organic frameworks: gas sorption isotherms for Zn(BDC) (BDC=1,4-benzenedicarboxylate). J. Am. Chem. Soc. 1998, 120, 8571-8572.

Li, B.; Cong, R.; Luo, Y.; Tan, B. Tailoring the pore size of hypercrosslinked polymers. Soft. Matter. 2011, 7, 10910-10916.

Li, J.; Yang, X.; Bai, C.; Tian, Y.; Li, B.; Zhang, S.; Yang, X.; Ding, S.; Xia, C.; Tan, X.; Ma, L.; Li, S. A novel benzimidazole-functionalized 2-D COF material: Synthesis and application as a selective solid-phase extractant for separation of uranium. J. Colloid Interf. Sci. 2015, 437, $211-218$.

Li, Z.; Zhang, Y.; Xia, H.; Mu, Y.; Liu, X. A robust and luminescent covalent organic framework as a highly sensitive and selective sensor for the detection of Cu2+ ions. Chem. Commun. 2016, 52, 6613-6616.

Lin, G.; Ding, H.; Yuan, D.; Wang, B.; Wang, C. A pyrene-based, fluorescent three-dimensional covalent organic framework. J. Am. Chem. Soc. 2016, 138, 3302-3305.

Lin, G.; Ding, H.; Chen, R.; Peng, Z.; Wang, B.; Wang, C. 3D porphyrin-based covalent organic frameworks. J. Am. Chem. Soc. 2017, 139, 87058709.

Liu, X. H.; Guan, C. Z.; Ding, S. Y.; Wang, W.; Yan, H. J.; Wang, D.; Wan, L. J. On-surface synthesis of single-layered two-dimensional covalent organic frameworks via solid-vapor interface reactions. J. Am. Chem. Soc. 2013, 135, 10470-10474.

Liu, J.; Cao, J.; Chen, H.; Zhou, D. Adsorptive removal of humic acid from aqueous solution by micro- and mesoporous covalent triazine-based framework. Colloids Surf. A 2015, 481, 276-282.

Luo, Y.; Li, B.; Wang, W.; Wu, K.; Tan, B. Hypercrosslinked aromatic heterocyclic microporous polymers: a new class of highly selective $\mathrm{CO}_{2}$ capturing materials. Adv. Mater. 2012, 24, 5703-5707.

Ma, T.; Kapustin, E. A.; Yin, S. X.; Liang, L.; Zhou, Z.; Niu, J.; Li, L. H.; Wang, Y.; Su, J.; Li, J.; Wang, X.; Wang, W. D.; Wang, W.; Sun, J.; Yaghi, O. M. Single-crystal x-ray diffraction structures of covalent organic frameworks. Science 2018, 361, 48-52. DOI: 10.1126/science.aat7679.

Manecke, V. G.; Wöhrle, D. Synthese und halbleitereigenschaften einiger komplexe und der aus ihnen hergestellten polymeren. Teil 2. Polymere mit phthalocyaninartiger und triazinartiger stuktur. Die Makromolekulare Chemie 1968, 120, 176-191.

McKeown, N. B.; Budd, P. M. Polymers of intrinsic microporosity (PIMs): organic materials for membrane separations, heterogeneous catalysis and hydrogen storage. Chem. Soc. Rev. 2006, 35, 675-683.

Medina, D. D.; Werner, V.; Auras, F.; Tautz, R.; Dogru, M.; Schuster, J.; Linke, S.; Döblinger, M.; Feldmann, J.; Knochel, P.; Bein, T. Oriented thin films of a benzodithiophene covalent organic framework. ACS Nano 2014, 8, 4042-4052.

Nagai, A.; Chen, X.; Feng, X.; Ding, X.; Guo, Z.; Jiang, D. A squaraine-linked mesoporous covalent organic framework. Angew Chem. Int. Ed. 2013, 52, 3770-3774.

Niu, X.; Ding, S.; Wang, W.; Xua, Y.; Xu, Y.; Chen, H.; Chen, X. Separation of small organic molecules using covalent organic frameworks-LZU1 as stationary phase by open-tubular capillary electrochromatography. J. Chromatogr. A 2016, 1436, 109-117.

Plas, J.; Ivasenko, O.; Martsinovich, N.; Lackinger, M.; De Feyter, S. Nanopatterning of a covalent organic framework host-guest system. Chem. Commun. 2015, 52, 68-71.

Qian, C.; Qi, Q. Y.; Jiang, G. F.; Cui, F. Z.; Tian, Y.; Zhao, X. Toward covalent organic frameworks bearing three different kinds of pores: the strategy for construction and COF-to-COF transformation via heterogeneous linker exchange. J. Am. Chem. Soc. 2017, 139, 6736-6743.

Rao, X.; Song, T.; Gao, J.; Cui, Y.; Yang, Y.; Wu, C.; Chen, B.; Qian, G. A highly sensitive mixed lanthanide metal-organic framework selfcalibrated luminescent thermometer. J. Am. Chem. Soc. 2013, 135, 15559-15564.

Salonen, L. M.; Medina, D. D.; Carbó-Argibay, E.; Goesten, M. G.; Mafra, L.; Guldris, N.; Rotter, J. M.; Stroppa, D. G.; Rodríguez-Abreu, C. A supramolecular strategy based on molecular dipole moments for high-quality covalent organic frameworks. Chem. Commun. 2016, 52, 7986-7989.

Sublemontier, O.; Nicolas, C.; Aureau, D.; Patanen, M.; Kintz, H.; Liu, X.; Gaveau, M. A.; Garrec, J. L. L.; Robert, E.; Barreda, F. A.; Etcheberry, A.; Reynaud, C.; Mitchell, J. B.; Miron, C. X-ray photoelectron spectroscopy of isolated nanoparticles. J. Phys. Chem. Lett. 2014, 5, 3399-3403.

Spitler, E. L.; Dichtel, W. R. Lewis acid-catalysed formation of two-dimensional phthalocyanine covalent organic frameworks. Nat. Chem. 2010, 2, 672-677.

Sumida, K.; Rogow, D. L.; Mason, J. A.; McDonald, T. M.; Bloch, E. D.; Herm, Z. R.; Bae, T. H.; Long, J. R. Carbon dioxide capture in metalorganic frameworks. Chem. Rev. 2012, 112, 724-781.

Waller, P. J.; Gandara, F.; Yaghi, O. M. Chemistry of covalent organic frameworks. Accounts Chem. Res. 2015, 48, 3053-3063.

Wan, S.; Guo, J.; Kim, J.; Ihee, H.; Jiang, D. A belt-shaped, blue luminescent, and semiconducting covalent organic framework. Angew. Chem. Int. Ed. 2008, 47, 8826-8830.

Wan, S.; Gandara, F.; Asano, A.; Furukawa, H.; Saeki, A.; Dey, S. K.; Liao, L.; Ambrogio, M. W.; Botros, Y. Y.; Duan, X.; Seki, S.; Stoddart, J. F.; Yaghi, O. M. Covalent organic frameworks with high charge carrier mobility. Chem. Mater. 2011, 23, 4094-4097.

Wang, T.; Kailasam, K.; Xiao, P.; Chen, G.; Chen, L.; Wang, L.; Li, J.; Zhu, J. Adsorption removal of organic dyes on covalent triazine framework (CTF). Micropor. Mesopor. Mater. 2014a, 187, 63-70.

Wang, X. S.; Chrzanowski, M.; Yuan, D.; Sweeting, B. S.; Ma, S. Shape-controlled nanofabrication of conducting polymer on planar DNA templates. Chem. Mater. 2014b, 26, 1639-1644.

Wang, W.; Wang, J.; Zhang, S.; Cui, P.; Wang, C.; Wang, A. novel Schiff base network-1 nanocomposite coated fiber for solid-phase microextraction of phenols from honey samples. Z. Talanta 2016a, 161, 22-30.

Wang, Y.; Li, J.; Yang, Q.; Zhong, C. Two-dimensional covalent triazine framework membrane for helium separation and hydrogen purification. ACS Appl. Mater. Interfaces 2016b, 8, 8694-8701.

Wang, L. L.; Yang, C. X.; Yan, X. P. Exploring fluorescent covalent organic frameworks for selective sensing of Fe ${ }^{3+}$. Sci. China. Chem. 2018, 61, 1470-1474. 
Wu, M.; Chen, G.; Ma, J.; Liu, P.; Jia, Q. Fabrication of cross-linked hydrazone covalent organic frameworks by click chemistry and application to solid phase microextraction. Talanta 2016, 161, 350-358.

Xie, Y.; Ding, S.; Liu, J.; Wang, W.; Zheng, Q. Triazatruxene based covalent organic framework and its quick-response fluorescence-on nature towards electron rich arenes. J. Mater. Chem. C. 2015, 3, 10066-10069.

Xu, H.; Chen, X.; Gao, J.; Addicoat, M.; Irle, S.; Jiang, D. Catalytic covalent organic frameworks via pore surface engineering. Chem. Commun. 2014, 50, 1292-1294.

Xue, R.; Cuo, H.; Wang, T.; Cong, L.; Wang, Y.; Ai, J.; Huang, D.; Chen, H.; Yang, W. Fluorescence properties and analytical applications of covalent organic frameworks. Anal. Methods 2017, 9, 3737-3750.

Yang, S. J.; Choi, J. Y.; Chae, H. K.; Cho, J. H.; Nahm, K. S.; Park, C. R. Preparation and enhanced hydrostability and hydrogen storage capacity of CNT@MOF-5 hybrid composite. Chem. Mater. 2009, 21, 1893-1897.

Zha, Z.; Xu, L.; Wang, Z.; Li, X.; Pan, Q.; Hu, P.; Lei, S. 3D graphene functionalized by covalent organic framework thin film as capacitive electrode in alkaline media. ACS Appl. Mater. Interfaces 2015, 7, 17837-17843.

Zhang, C.; Li, G.; Zhang, Z. A hydrazone covalent organic polymer based micro-solid phase extraction for online analysis of trace Sudan dyes in food samples. J. Chromatogr. A 2015, 1419, 1-9.

Zhang, S.; Zhao, X.; Li, B.; Bai, C.; Li, Y.; Wang, L.; Wen, R.; Zhang, M.; Ma, L.; Li, S. “Stereoscopic” 2D super-microporous phosphazene-based covalent organic framework: design, synthesis and selective sorption towards uranium at high acidic condition. J. Hazard. Mater. 2016a, 314, 95-104.

Zhang, Y. B.; Su, J.; Furukawa, H.; Yun, Y.; Gandara, F.; Duong, A.; Zou, X.; Yaghi, O. M. Single-crystal structure of a covalent organic framework. J. Am. Chem. Soc. 2013, 135, 16336-16339.

Zhang, Y.; Shen, X.; Feng, X.; Xia, H.; Mu, Y.; Liu, X. Covalent organic frameworks as pH responsive signaling scaffolds. Chem. Commun. 2016b, 52, 11088-11091.

Zhang, C.; Zhang, S.; Yan, Y.; Xia, F.; Huang, A.; Xian, Y. Highly fluorescent polyimide covalent organic nanosheets as sensing probes for the detection of 2,4,6-trinitrophenol. ACS Appl. Mater. Interfaces 2017a, 9, 13415-13421.

Zhang, S.; Yang, Q.; Li, Z.; Wang, W.; Wang, C.; Wang, Z. Covalent organic frameworks as a novel fiber coating for solid-phase microextraction of volatile benzene homologues. Anal. Bioanal. Chem. 2017b, 409, 3429-3439.

Zhang, W.; Qiu, L. G.; Yuan, Y. P.; Xie, A. J.; Shen, Y. H.; Zhu, J. F. Microwave-assisted synthesis of highly fluorescent nanoparticles of a melamine-based porous covalent organic framework for trace-level detection of nitroaromatic explosives. J. Hazard. Mater. 2012, 221$222,147-154$.

Zhang, Y. B.; Su, J.; Furukawa, H.; Yun, Y.; Gandara, F.; Duong, A.; Zou, X.; Yaghi, O. M. Single-crystal structure of a covalent organic framework. J. Am. Chem. Soc. 2013, 135, 16336-16339.

Zhou, T. Y.; Xu, S. Q.; Wen, Q.; Pang, Z. F.; Zhao, X. One-step construction of two different kinds of pores in a 2D covalent organic framework. J. Am. Chem. Soc. 2014, 136, 15885-15888.

Zou, X.; Ren, H.; Zhu, G. Topology-directed design of porous organic frameworks and their advanced applications. Chem. Commun. 2013, 49, 3925-3936. 